\title{
بناء اختبار محكي المرجع لقياس العمليات الأسـاسية في مادة الرياضيات لخريجات المرحلة الابتدائية باستخدام نظرية استجابة الفقرة
}

\author{
عائشسة محمد صيايل العنزي \\ باحثة- المملكة العربية السعودية
}

صبري محمد إسماعيل عبدالعال

أستاذ مساعد في القياس والتقويم التربوي- كلية التربية والآداب جامعة تبوك- المملكة العربية السعودية

sabdelaal@ut.edu.sa

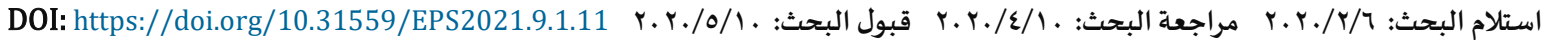

هدفت هذه الدِّراسـة إلى بناء اختبار محكي المرجع؛ لقياس العمليَّات الأساسيَّة في الرِِّاضيَّات وفق نظريَّة استجابة الفقرة، حيث تهَّ

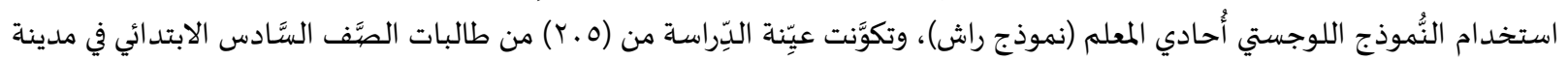

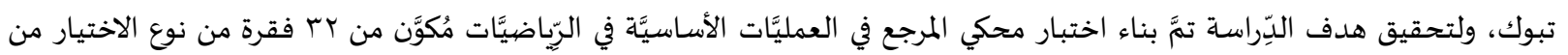

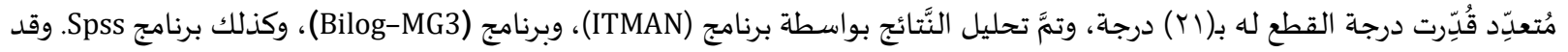

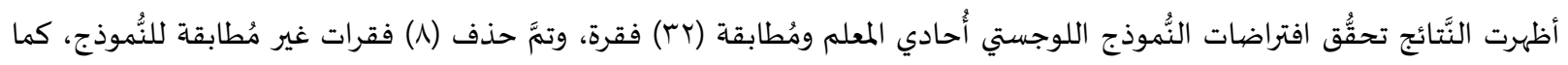

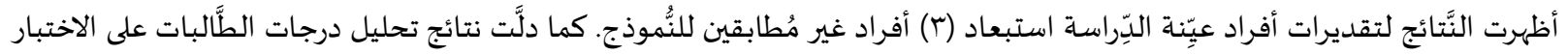

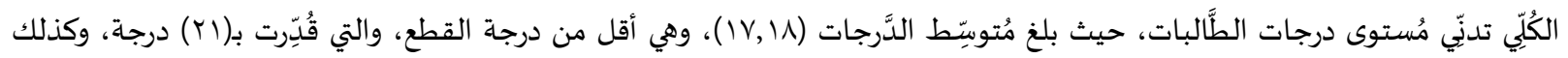

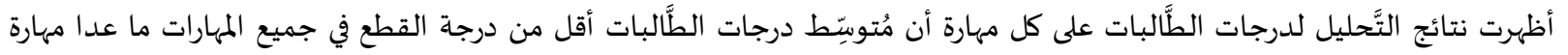

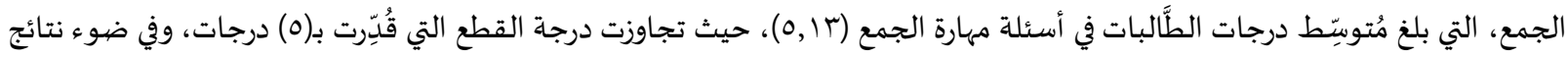

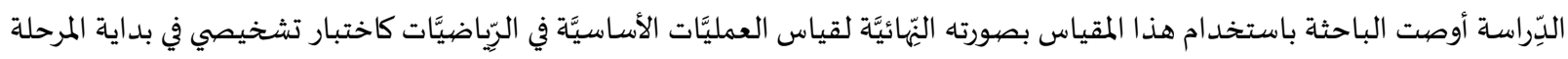

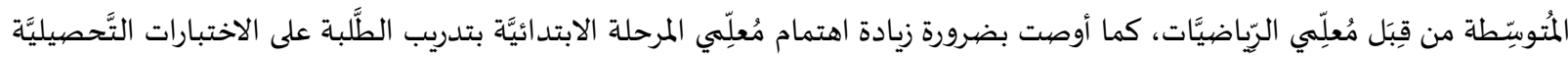

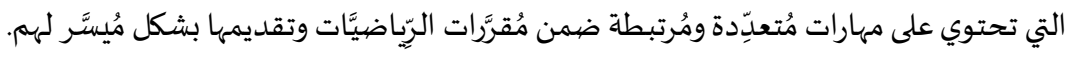
الكلمات المفتاحية: بناء اختبار؛ محكي المرجع؛ العمليَّات الأساسيَّة؛ نظريَّة استجابة الفقرة.

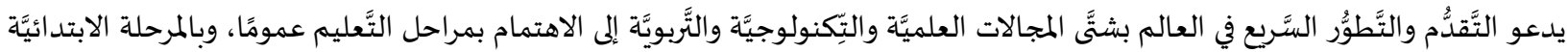

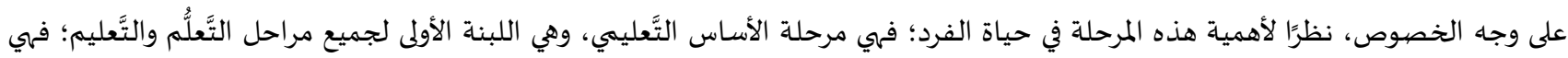

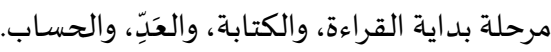

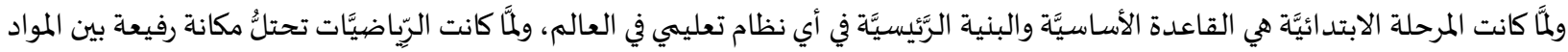

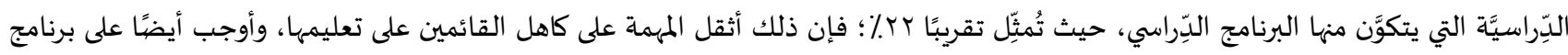

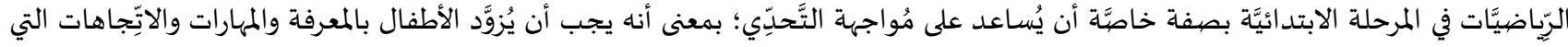

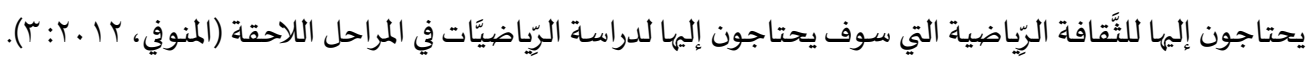




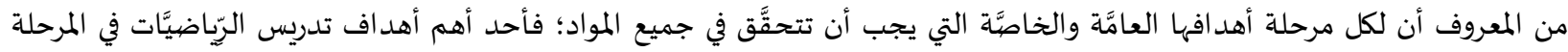

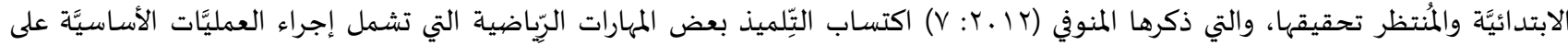

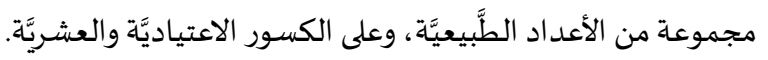

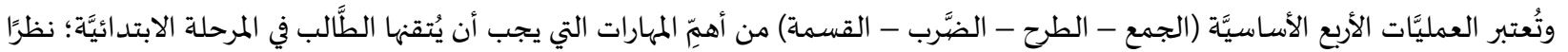

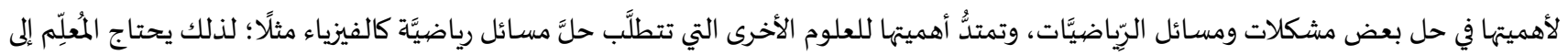

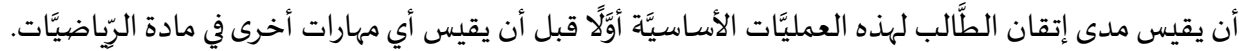

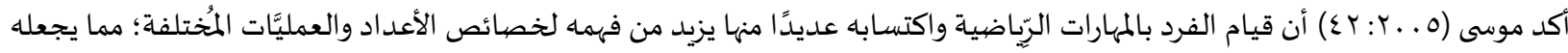

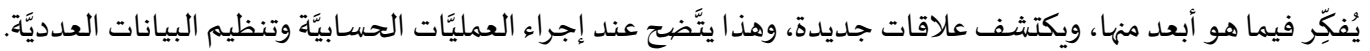

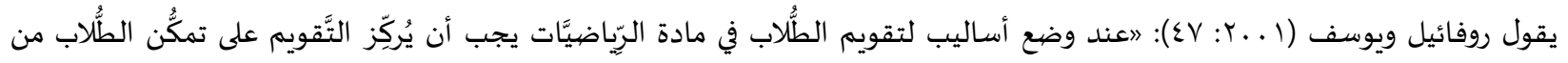

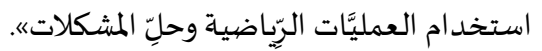

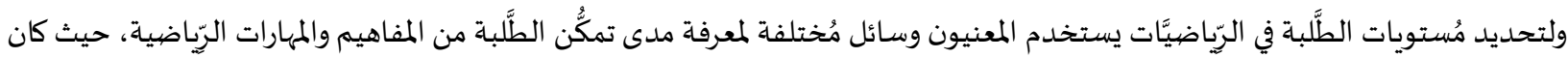

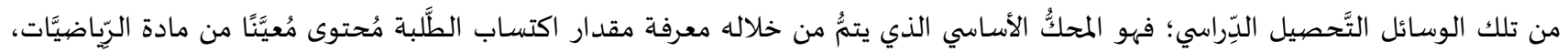

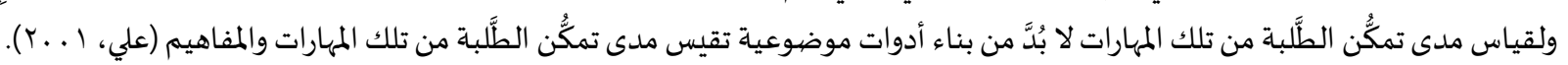

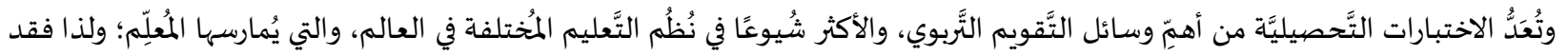

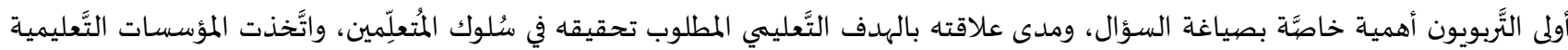

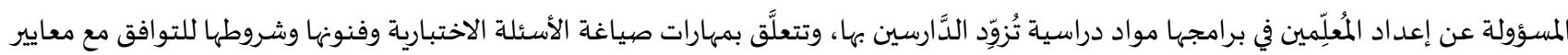

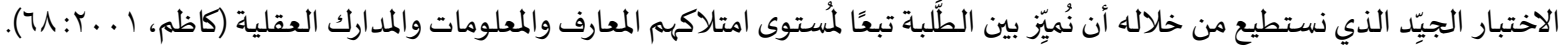

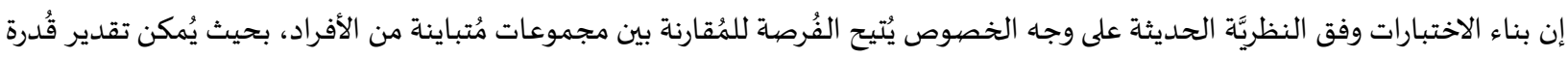

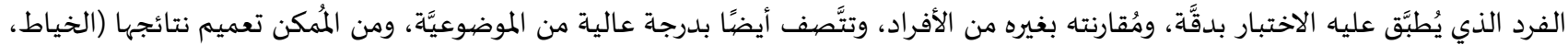

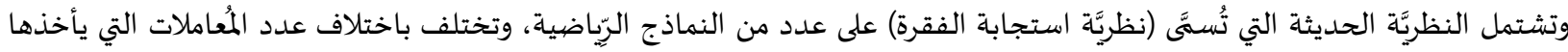

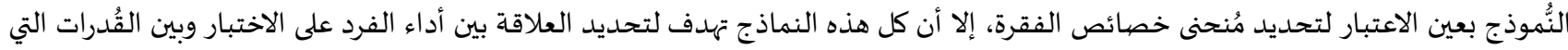

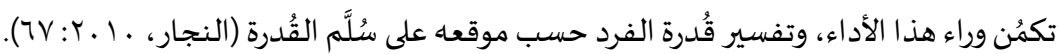

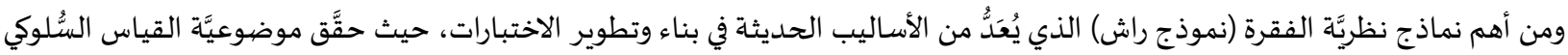

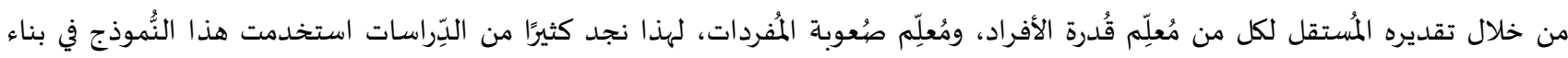

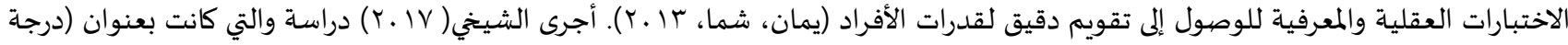

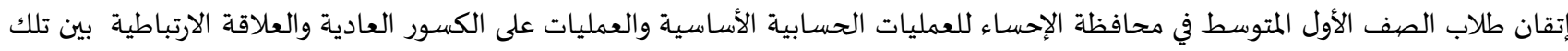

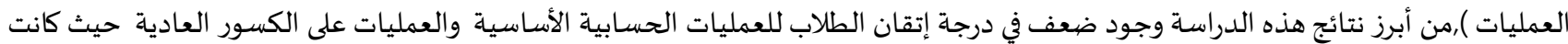

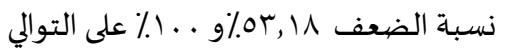

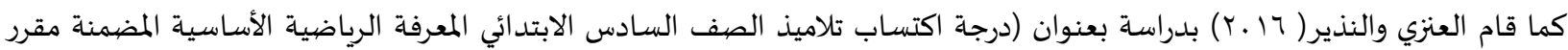
الرياضيات ) والتي هدفت إلى معرفة درجة اكتساب تلاميذ الصفف السادس الابتدائي المعرفة الرياضية الأساسية (مفاهيم - مهارات ) حيث تم بناء

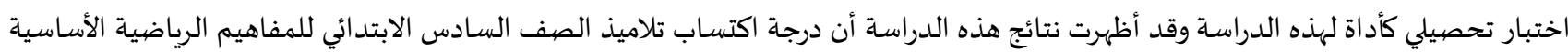

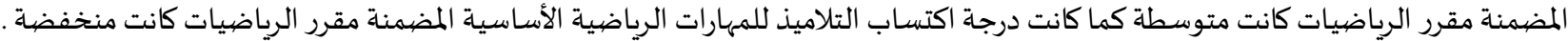

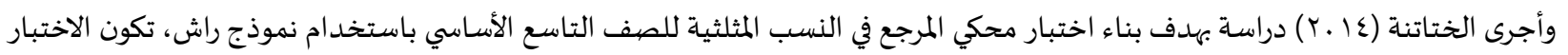

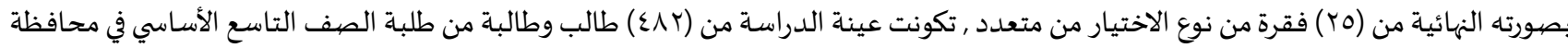

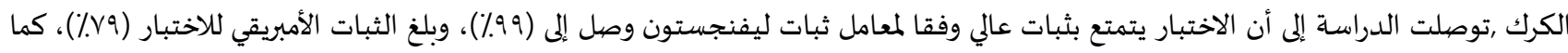

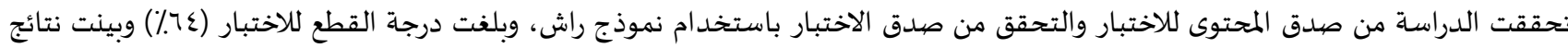

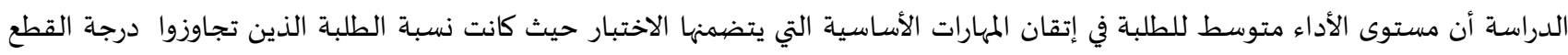

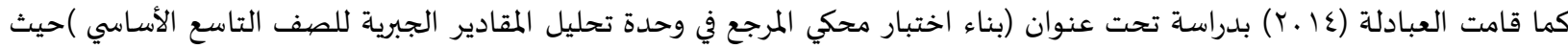

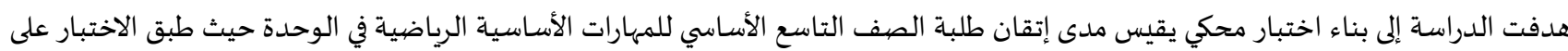




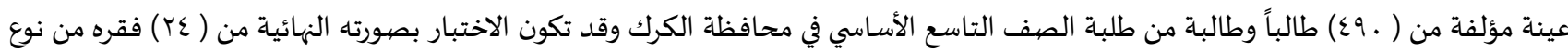

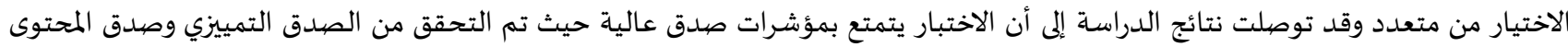

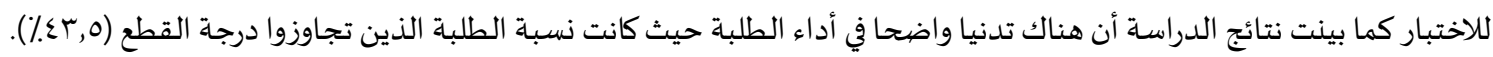

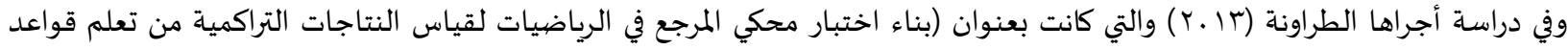

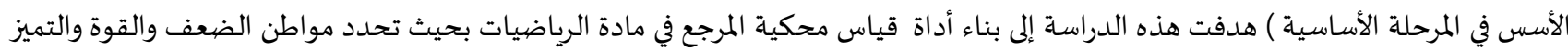

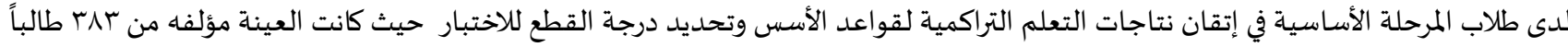

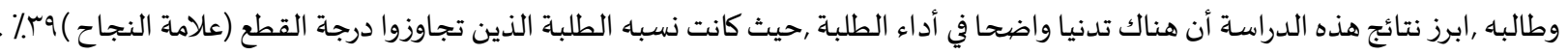

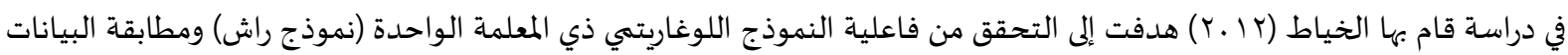

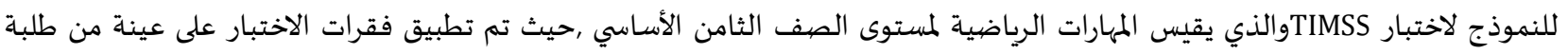

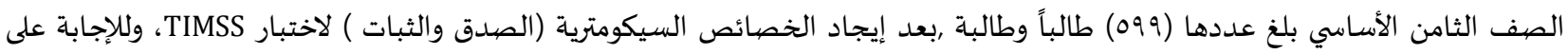

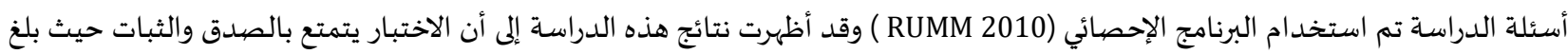

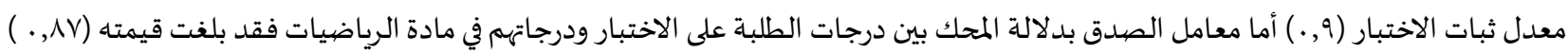

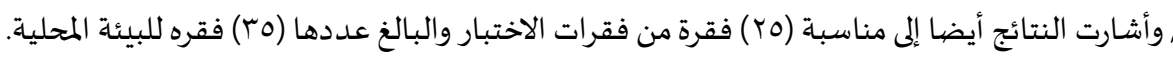

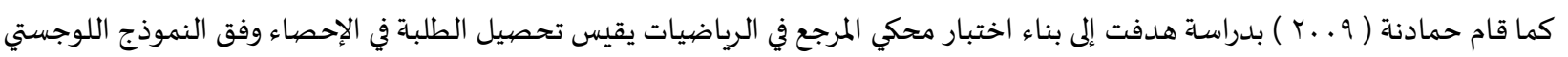

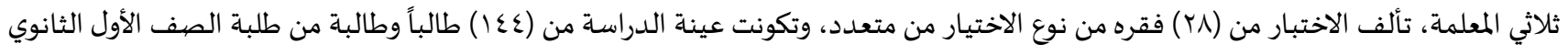

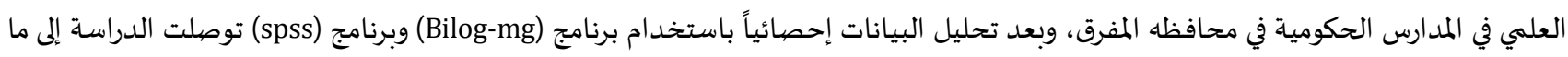

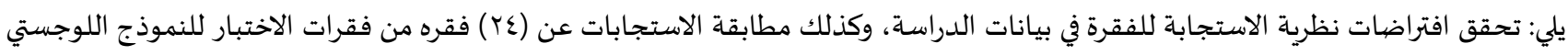

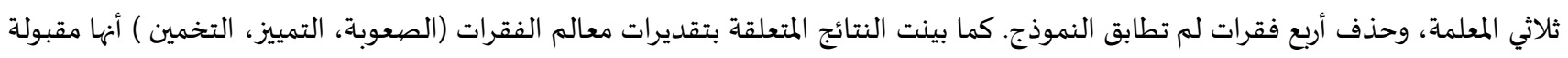
ضمن المحكات التي أوردها القياس التربوي. وفي دراسة ويلسون (Wilson, 2007) والتي هدفت لقياس المهارات الرياضية باستخدام اختبار موضيوعي (اختيار من متعدد) تكونت عينة

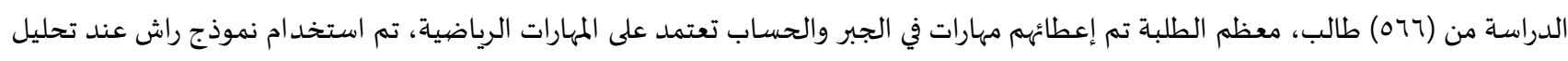

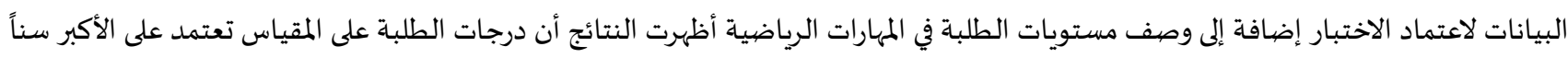

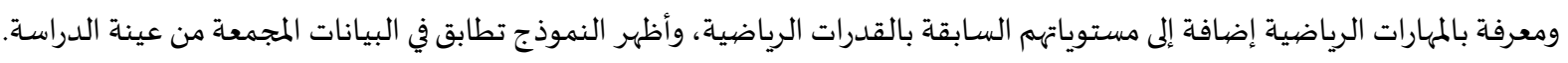

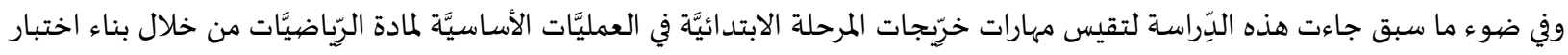

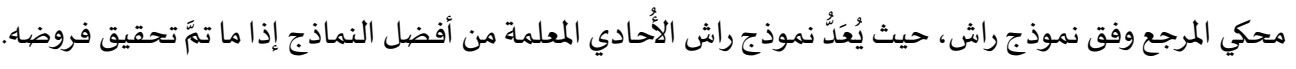

مُشَكلة الدِّراسة:

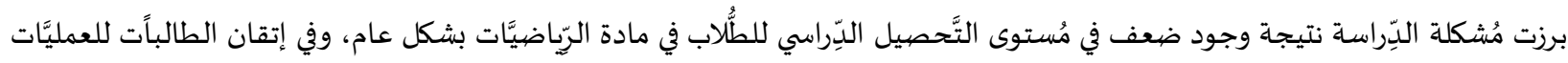

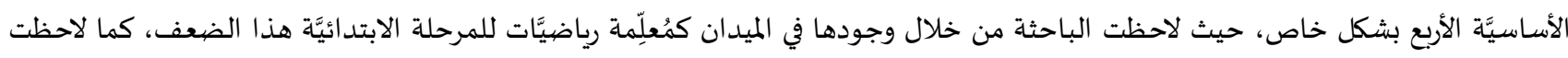

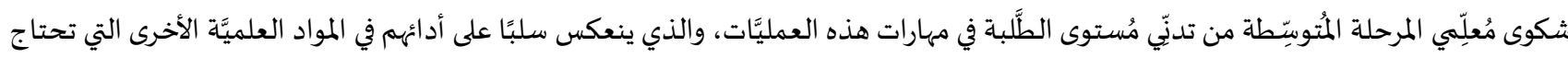

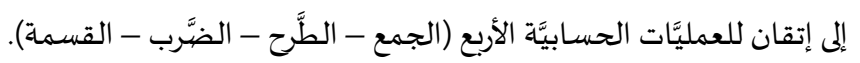

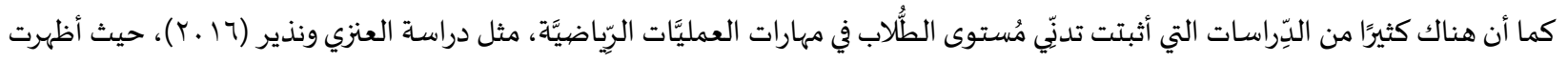

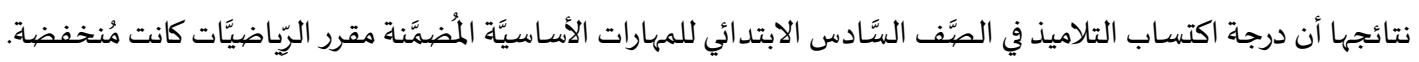

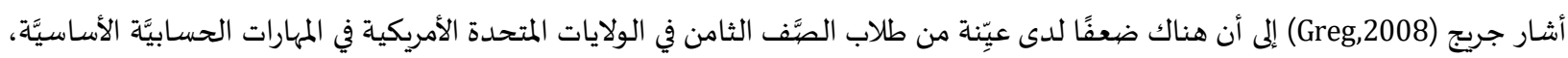

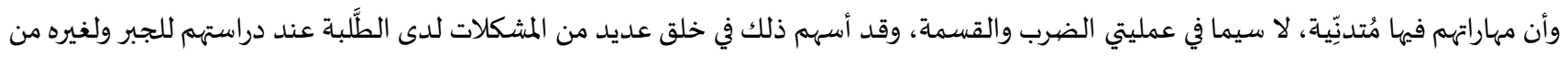

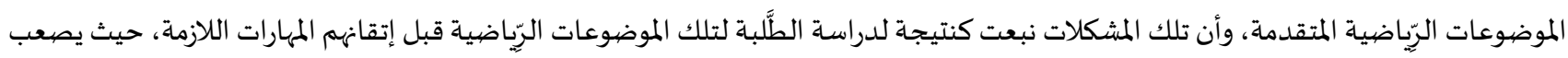

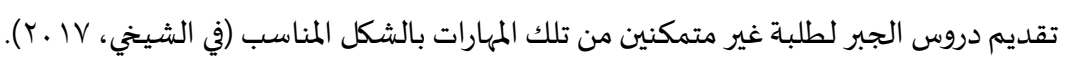

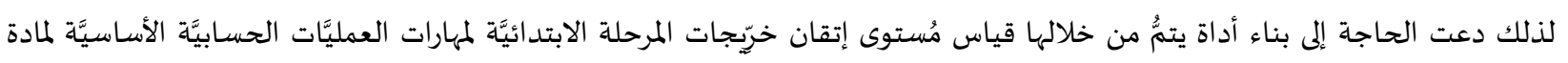
الرِّاضيَّات. 
أسئلة الدِّراسـة:

السُّؤال الأوَّلّ: ما مدلى تحقُّق افتراضات نموذج راش كأحد نماذج استجابة الفقرة؟

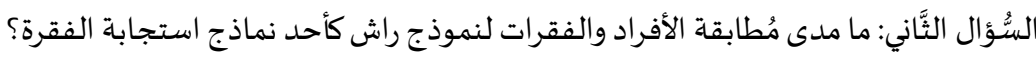

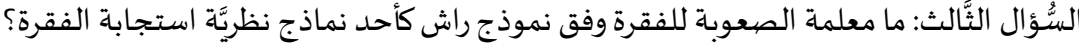

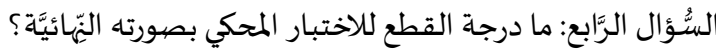

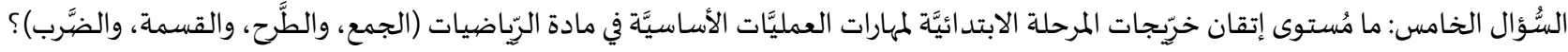
أهداف الدِّراسـة: ا. بناء اختبار محكي المرجع يقيس مُستوى إتقان خريجات المرحلة الابتدائَّة لمهارات العمليَّات الأسـاسيَّة في مادة الرِِّاضيَّات باستخدام نظريَّة استجابة الفقرة وفق نموذج راش.

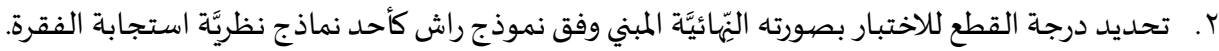

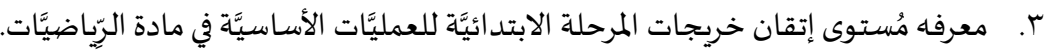

\section{أهمية الدِّراسـة:}

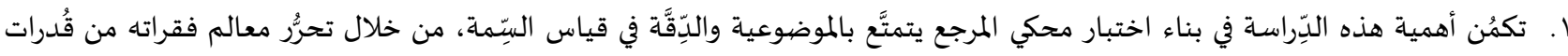

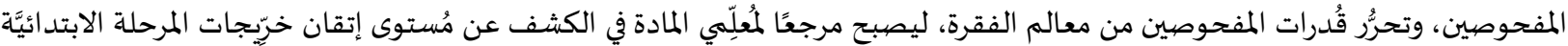

لبعمليَّات الأسـاسيَّة.

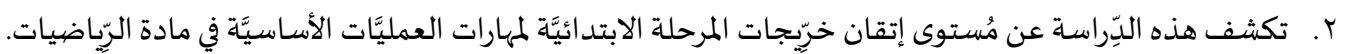

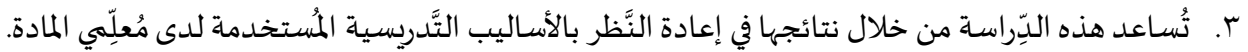

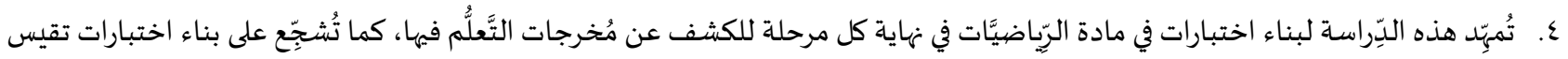

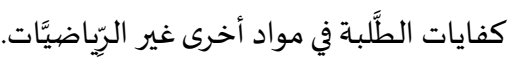

حدود الدراسـة: حدود ديموغر افيَّة: اقتصرت الدِّراسة على طالباًت الصيَّف السَّادس في المدارس الحكومية التابعة لإدارة التَّعليم في مدينة تبوك.

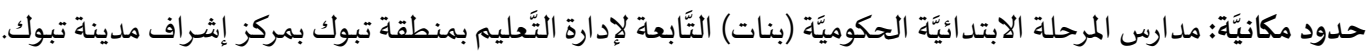

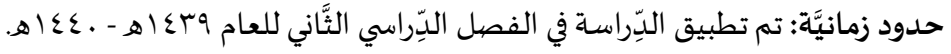

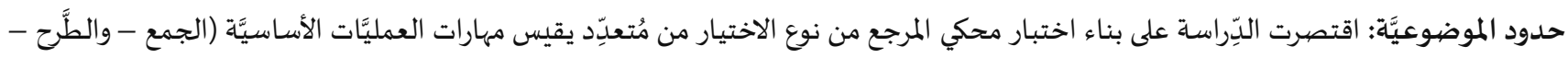

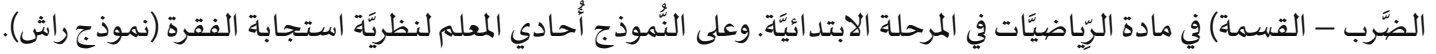

مُصبطلحات الدِّراسـة:

الاختبار المحكي المرجع: هو الاختبار الذي يُستخدم في تقييم أداء الفرد بالنِّسبة إلى محك (مُستوى أداء مُطلق) دون الحاجة إلى مُقارنة أد ائه بأداء الأفراد

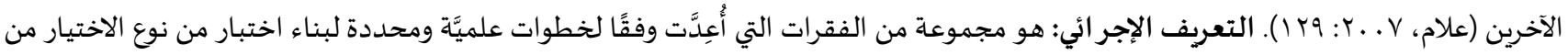

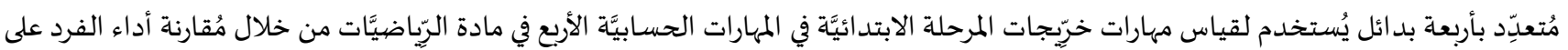

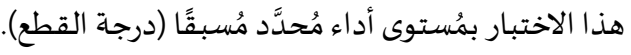

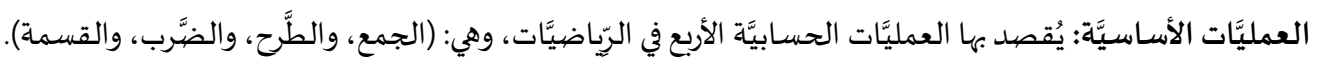

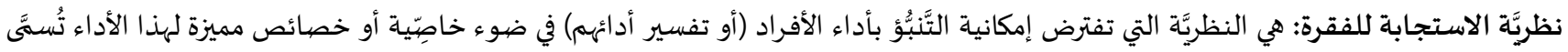

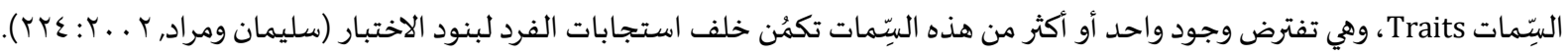

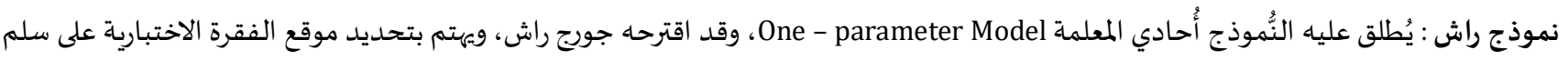
صعوبة لجميع الفقرات التي تشكل الاختبار، كما يهتم بتدريج مُستويات قُدرة الفرد باختبار معين على نفس متصل المعل الفقرات (علام، 10 ـ ب: 799). مُجتمع الدِّراسـة:

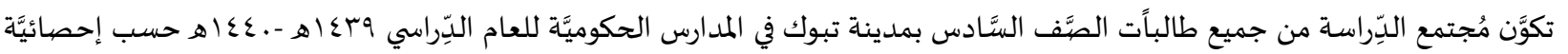

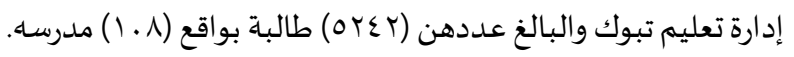


عِيّنة الدِّراسـة:

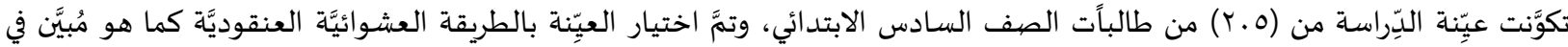

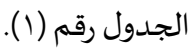

جدول (1): عينة الدراسـة

\begin{tabular}{|c|c|c|c|}
\hline النِّبسبة المئوية من العِّنَة الكلية & 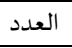 & اسم المدرسة & م \\
\hline$\% \cdot, v r$ & rt & أروى بن أنيس & 1 \\
\hline$\% 11$ & $r v$ & الابتدائيَّة الثَّامنة عشرة & r \\
\hline$\% 1,90$ & $\varepsilon_{0}$ & الابتدائيَّة التَّاسعة عشرة & r \\
\hline$\%$ \%q & 1.1 & الابتدائيَّة الثَّالثة والثَّلاثون & $\varepsilon$ \\
\hline
\end{tabular}

منهجبيَّة الدِّراسـة:

اعتمد في هذه الذِّراسة المنههج الوصفي التَّحليلي الذي يستخدم أسلوبًا مسحيَّا لجمع البيانات بواسطة اختبار محكي تمَّ تحليله إحصائيًّا بما يتَّفق

مع أسئلة وأهد اف الدِّراسة.

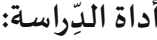

تمَّ بناء اختبار محكي المرجع يقيس مهارات طالباًت الصَّف السَّادس في العمليَّات الأساسيَّة (الجمع، والطَّرح، والضَّرب، والقسمة) في مادة الرِّّاضيَّات وفق نظرية استجابة الفقرة باستخدام نموذج راش، حيث تمَّت صياغة (0ع) فقرة من نوع الاختيار من مُتعدِّد بأربعة بدائل لكل فقرة، ولكل

فقرة إجابة واحدة صحيحة.

بناء الأداة:

تحديد الغرض من الاختبار وهو قياس مهارات الطالباًت في العمليَّات الأسـاسيَّة (الجمع - الطَّرح - الضَّرب - القسمة) في مادة الرِِّاضيَّات.

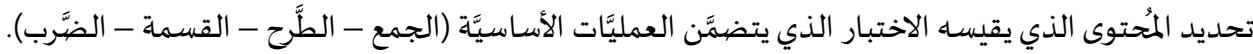

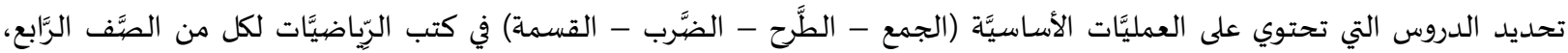
والخامس,والسَّادس الابتدائي للفصلين اللِّراسيين الأوَّل والثَّاني. صياغة الأهداف السلوكية لكل عملية من العمليَّات الأربع، وذلك بالاستعانة بمعلمات ومشرفات المادة مع مُراعاة حذف الأهداف المكررة والأهداف التي تحتوي مهارة غير أساسيَّة. بناء جدول مواصفات حسب عدد الأهداف ومُستوى كل هدف. بناء فقرات الاختبار استنادًا إلى الأهد اف السلوكية التي تمَّ إعد ادها، حيث اشتمل الاختبار في صورته الأوَّلية على ه؛ فقرة من فقرات الاختيار من

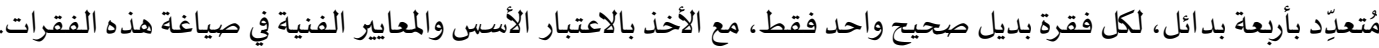
تمَّ عرض الاختبار في صورته الأولية على عدد من المحكمين من أعضاء هيئة التدريس في تخصص القياس والتقويم وتخصص مناهج وطرق

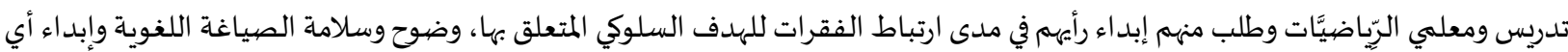
ملاحظات يرونها مناسبة من خلال الاستبيان المعد للتحكيم. تمَّ الأخذ بآراء المحكمين، حيث قدموا مجموعة من الاقتراحات والملاحظات التي بناءً عليها جرى تعديل بعض الفقرات بحيث يصبح الاختبار جاهزًا للتطبيق الميداني. إعداد ورقة تعليمات للاختبار وورقة نموذج الإجابة. التجريب والتطبيق الأوَّلي للاختبار:

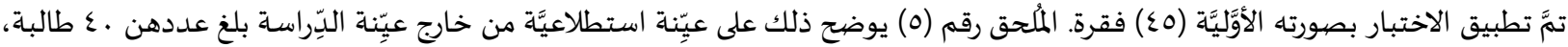

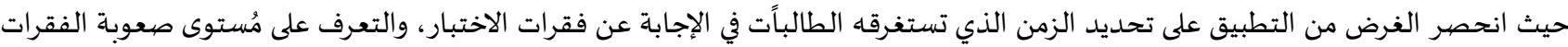

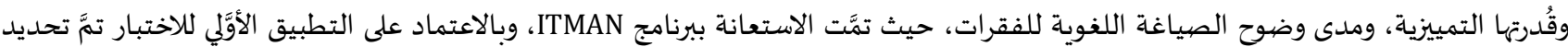
الزمن المناسب للاختبار بواقع (0ء) دقيقة، وتم حساب مُعاملات الصعوبة والتمييز للفقرات كما في الجدول رقم (؟). 
جدول (Y): مُعاملات الصعوبة والتمييزلفقرات الاختبار للعينة الاستطلاعية

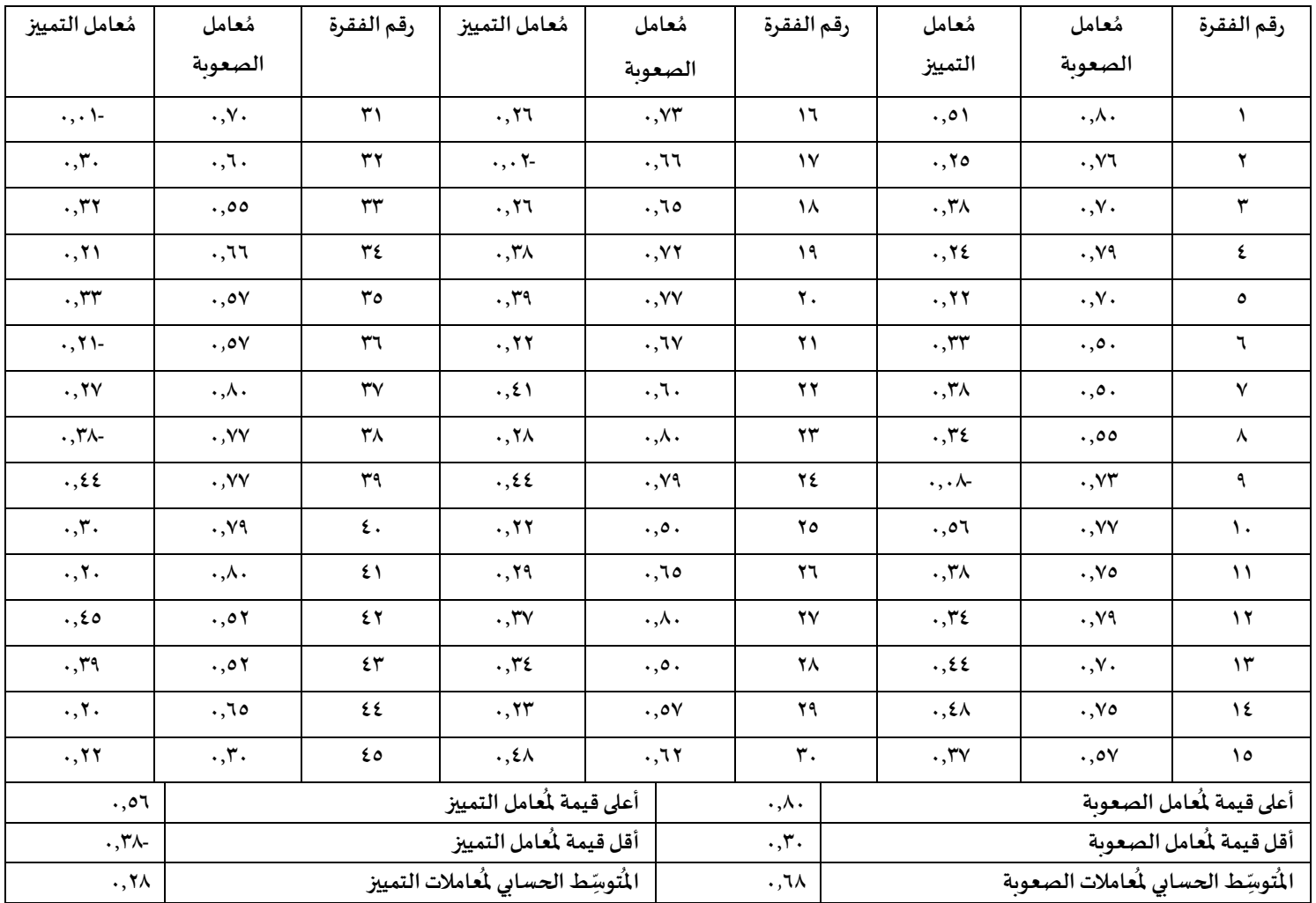

يتَّضح من الجدول رقم (r) أن مُعاملات الصعوبة في نموذج الاختبار محكي المرجع للعيّنة الاستطلاعية تراوحت بين (.r,. و .ی,..) بمُتوسِّط

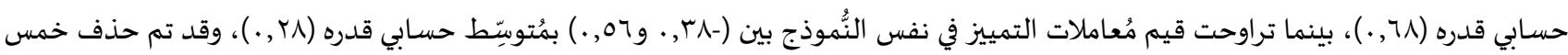

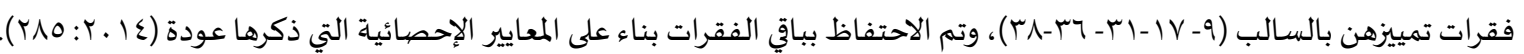

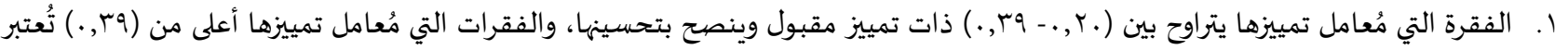

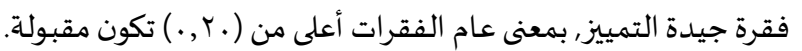

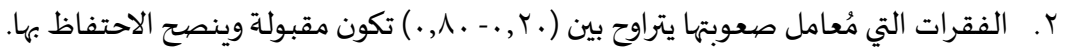

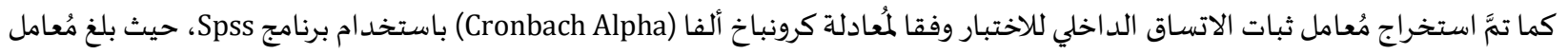
الثبات ا و, .، وهذا يعني أن أداة الدِّراسة تتمتع بدرجة عالية من الثبات. تطبيق الاختباربصورته النِهِائيَّة (جمع البيانات):

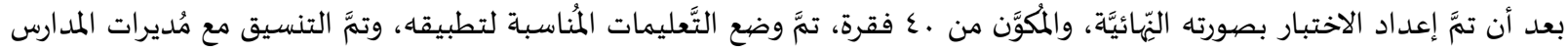
ومُعلِّمات المادة لتحديد موعد وآلية تطبيق الاختبار.

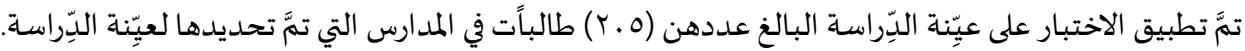

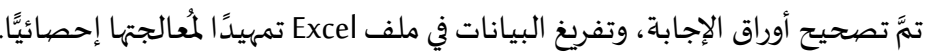
صيدق وثبات الأداة:

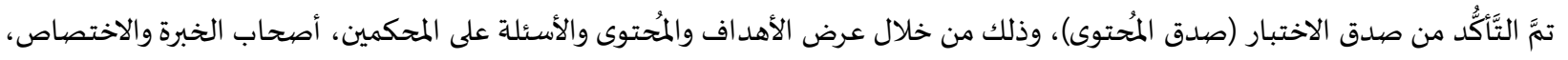

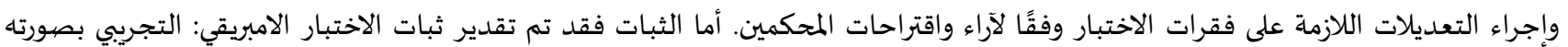

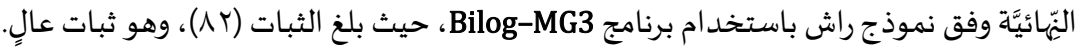

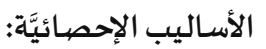
التَّحليل العاملي الاستكشافي للكشف عن أُحادية البُعد بواسطة برنامج الspss. استخراج مُعاملات الصعوبة والتمييز للعيّنة الاستطلاعيَّة باستخدام برنامج (ITMMAN). استخراج مُعامل الصعوبة وفقًا نموذج راش لنظريَّة استجابة الفقرة باستخدام برنامج Bilog-MG3. 
حساب مُعامل ثبات الاتساق الداخلي للاختبار وفقًا لمُعادلة كرونباخ ألفا (Cronbach Alpha) باستخدام برنامج Spss،، وحساب مُعامل الثبات

الامبريقي بواسطة برنامج Bilog-MG3.

استخدام مربع كاي لمُطابقة بيانات عيّنة البحث مع نموذج راش.

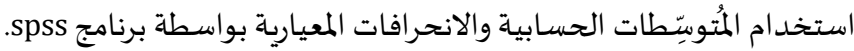

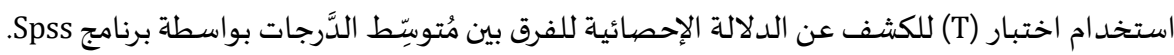

النتائج وتفسيرها:

السُّؤال الأوَّل: ما مدى تحقُّق افتراضـات نموذج راش كأحد نماذج استجابة الفقرة؟ ا

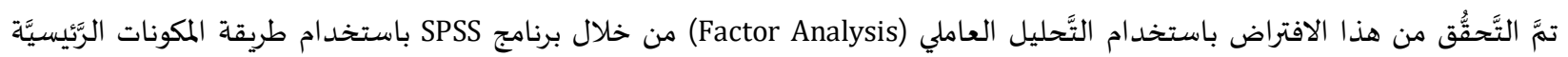

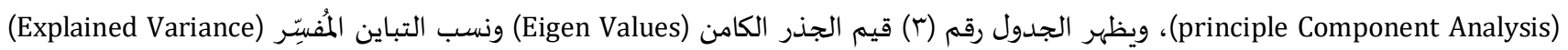
للعوامل الأربعة الأولى وناتج قسمة الجذر الأوَّل على العامل الثَّاني.

\begin{tabular}{|c|c|c|c|c|}
\hline الجذر الكامن الثاني & 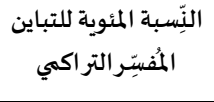 & التباينِ المُفِيّرِ المئوية & الجذر الكامن & العامل \\
\hline \multirow[t]{3}{*}{$r, r . q$} & $r I, V \varepsilon 1$ & $r \mid, V \varepsilon 1$ & 1,797 & الأوَّلَ \\
\hline & $\lceil\Lambda, 017$ & $7, \mathrm{~V} V 0$ & r,VI. & الثَّاني \\
\hline & $r \varepsilon, \varepsilon .0$ & 0,119 & T, T०7 & الثًالث \\
\hline
\end{tabular}

نُلاحظ من الجدول رقم (ץ) أن قيمة التباين المُفسِّر للعامل الأوَّل تخطَّت ال. ب\% كمؤشر على أُحادية البُعد، كما أن قسمة الجذر الكامن للعامل

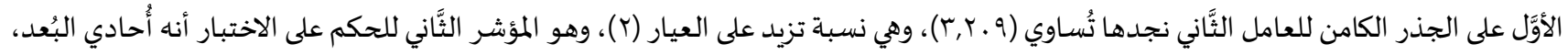

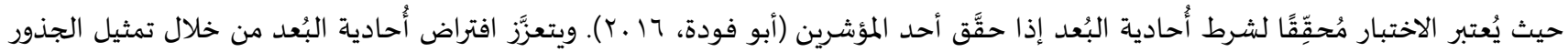
الكامنة بيانيًا باستخدام برنامج (SPSS) بما يُعرف باختبار فحص العوامل (Scree Plot) أو مخطط سكري، الذي يظهر في الشكل رقم (1)، والذي بيَّن

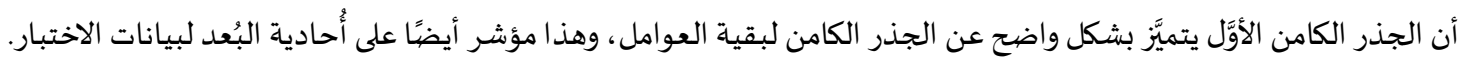

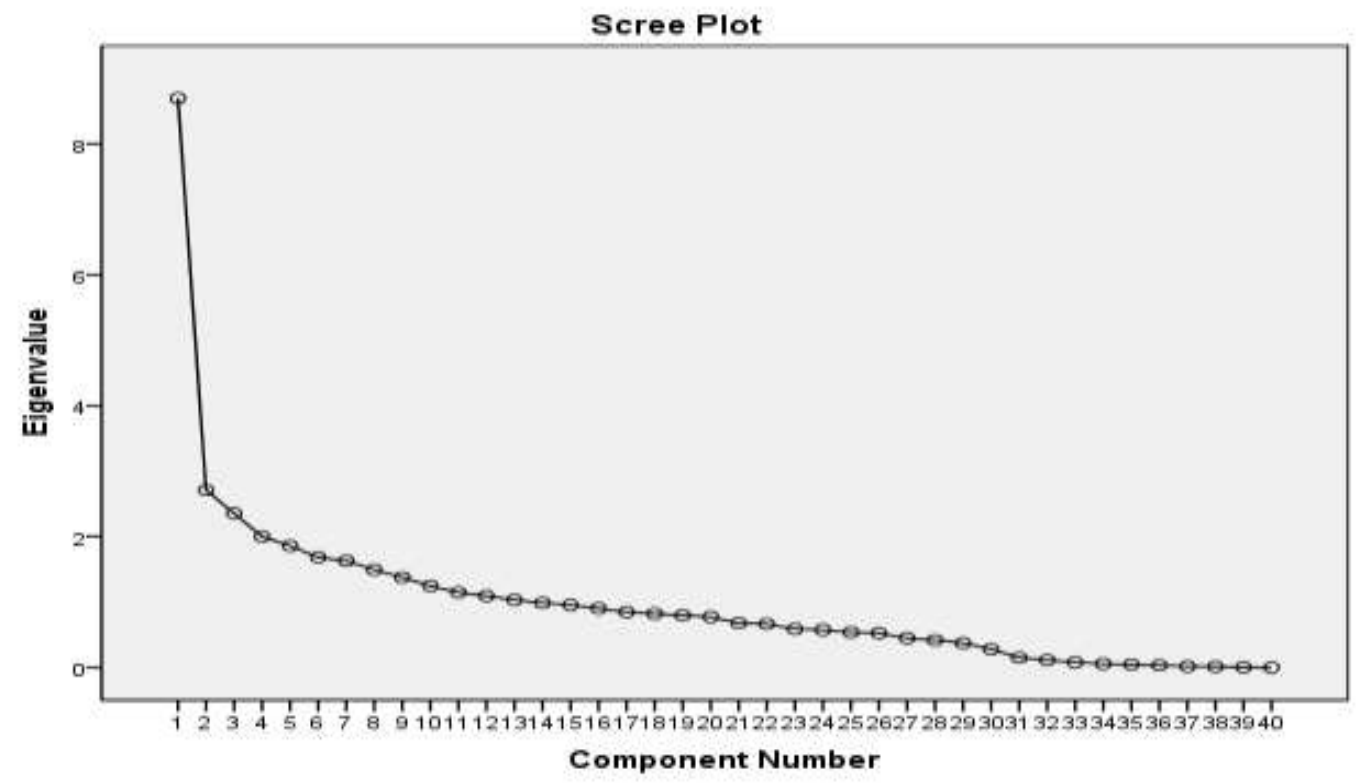

شكل (1): مخطط سكري لقيم الجذور الكامنة للعوامل المُستخخلَصهة من التَّحليل العاملي 


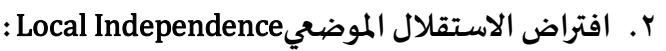

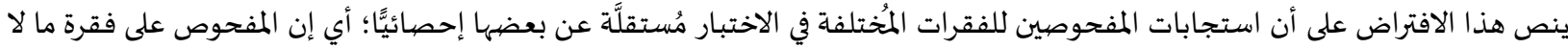

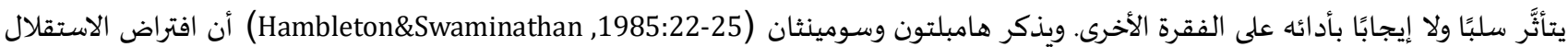

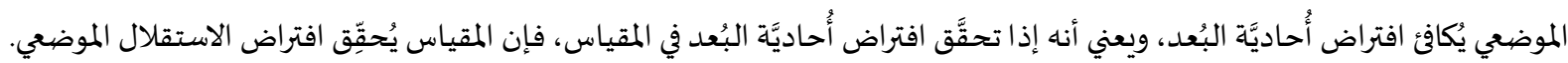

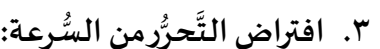

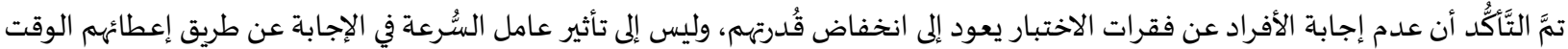
الكافي لهم أثناء تطبيق الاختبار.

السيُؤال الثَّاني: ما مدى مُطابقة الأفراد والفقرات لنموذج راش كأحد نماذج استجابة الفقرة؟ .

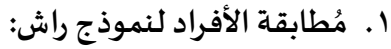

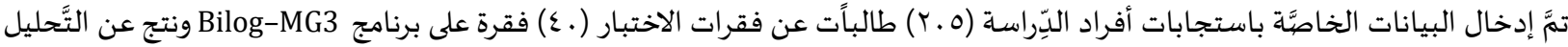

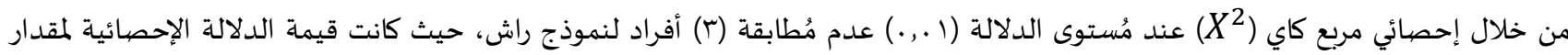

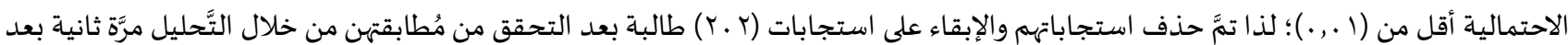

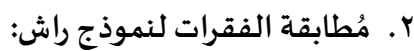

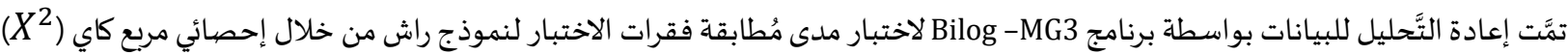

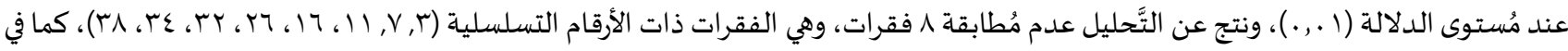

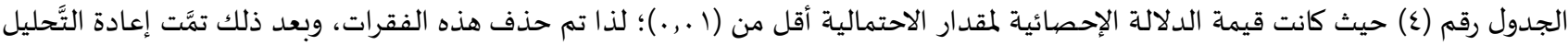
ونتج عن ذلك مُطابقة جميع الفقرات المتبقية (rr) فقرة لنموذج راش.

جدول (ع): مُطابقة فقرات الاختبار لنموذج راش الناب

\begin{tabular}{|c|c|c|c|c|c|}
\hline مُستوى الدلالة & قيمة (X) & رقم الفقرة & مُستوى الدلالة & قيمة (X) & رقم الفقرة \\
\hline .,9rq & $1, r$ & rI & , $\vee 9 \varepsilon$ & $r, \varepsilon$ & 1 \\
\hline .,07V & $\varepsilon, \Lambda$ & r &., $9 \vee$. & $1, r$ & $r$ \\
\hline., 947 & 1,1 & $r$ & $\ldots r$ & $1 \Lambda, 7$ & $r$ \\
\hline , AVY & $1, \Lambda$ & $T \varepsilon$ &., 109 & 7,7 & $\varepsilon$ \\
\hline .,700 & $r, r$ & ro &., ro9 & 7,0 & 0 \\
\hline$\cdot, \ldots \wedge$ & $1 \Lambda, \Lambda$ & r7 &.,$V \leqslant 7$ & $r, \mathrm{~V}$ & 7 \\
\hline . & 0,0 & TV &.,$\ldots r$ & $1 \Lambda, 7$ & v \\
\hline . & 7,1 & rA &., 117 & $\mathrm{~V}, \mathrm{o}$ & 1 \\
\hline., 190 & 7,1 & rq & ., YOV & $r, 7$ & 9 \\
\hline., $\mathrm{OVV}$ & $r, 9$ & $r$. &., 107 & 1,9 & 1. \\
\hline אזר, & $\varepsilon, \varepsilon$ & $r$ & $\cdot, \ldots v$ & $r u, r$ & 11 \\
\hline$\cdot, \ldots 7$ & $1 \wedge, 1$ & rr & . & $r, 7$ & Ir \\
\hline., 079 & $r, q$ & rT &., $9 \leq \varepsilon$ & $1, \mathrm{~V}$ & 14 \\
\hline.,..$\vee$ & 11,0 & $r \varepsilon$ &.,$Y V I$ & $7, \varepsilon$ & $1 \varepsilon$ \\
\hline .,.or & $1 ., 9$ & ro &., $9 Y \varepsilon$ & 1,9 & 10 \\
\hline$\cdot, 191$ & $\Lambda, 7$ & $r 7$ &.,$\ldots 1$ & $r \cdot, 0$ & 17 \\
\hline ., & $r, \Lambda$ & rV &., 104 & $\wedge,$. & IV \\
\hline.,$\ldots \wedge$ & 10,0 & ru &., 117 & $1 ., r$ & 11 \\
\hline . TrE & 0,1 & $r 9$ & $\cdot, \wedge 9 \vee$ & $r, r$ & 19 \\
\hline., 91. & $r, 1$ & $\varepsilon$. &., $1 \leqslant 7$ & 9,0 & $r$. \\
\hline
\end{tabular}

وبذلك تمَّ الحصيول على اختبار محكي المرجع يقيس مهارات العمليَّات الأسـاسيَّة في الرِّاضيَّات وفق نموذج راش يتكوَّن في صورته النِّهائَّة المُعتمدة 
السُّؤال الثَّالث: ما معلمة الصعوبة للفقرة وفق نموذج راش كأحد نماذج نظريَّة الفقرة؟ تم تقدير معلمة الصعوبة للفقرة والخطأ المعياري لتقدير المعلمة من خلال برنامج Bilog -MG3 كما في الجدول التالي.

جدول (0): قيم معلمة الصعوبة للفقرة والخطأ المعياري

\begin{tabular}{|c|c|c|c|c|c|}
\hline الخطأ المعياري للصعوبة & الصعوبة & رقم الفقرة & الخطأ المعياري للصعوبة & الصعوبة & رقم الفقرة \\
\hline$\cdot$, หา9 & $1,1.1-$ & IV & $\cdot, r \cdot r$ & T,YYA- & 1 \\
\hline$\cdot$, rרT &., $0 \wedge \varepsilon$ & 11 & $\cdot, r 7 r$ & $\cdot, \mathrm{V} 9 \mathrm{Y}$ & r \\
\hline$\cdot, r q$. & 1,091 & 19 & $\cdot, T \mid r$ & $r, \varepsilon \mid r-$ & $r$ \\
\hline 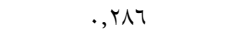 & $1, \Sigma 7 V$ & r. & $\cdot, r 70$ & $\cdot, 9 \wedge \varepsilon-$ & $\varepsilon$ \\
\hline.,$r M r$ & r,\&KI- & rI & $\cdot, \mathrm{YVI}$ &., $1 \cdot 1-$ & 0 \\
\hline ( ) & $r, \Sigma Y \wedge$ & rt & $\cdot, r \vee q$ & $1, \leqslant 70$ & 7 \\
\hline$\cdot, r \leqslant \Lambda$ & r,VVr & rt & $\cdot, r \cdot r$ & T,YYA- & v \\
\hline$\cdot, r 97$ & $1, \wedge Y 7$ & $r \varepsilon$ & $\cdot$, rרז & $\cdot, 9 \cdot 7-$ & $\wedge$ \\
\hline$\cdot$, rol & $1,00 \varepsilon$ & ro & $\cdot$, rol &., . ro & 9 \\
\hline., roג & 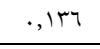 & ry & $\cdot, r 70$ & $\cdot, \mathrm{VV7}$ & 1. \\
\hline$\cdot, \mathrm{YM \Lambda}$ & $1,9 \varepsilon r-$ & TV &., 19 . & 1,01 . & 11 \\
\hline ., ror & $r, \wedge r \varepsilon$ & rᄉ & $\cdot, Y 7)$ & $\cdot, r r \varepsilon-$ & ir \\
\hline$\cdot, \mathrm{TV}$. &., $0 \wedge r$ & rq &., $\mathrm{rar}$ & $1,99 .-$ & $1 T$ \\
\hline ., rqV & $1,9 Y$. & $r$. & $., Y 7\}$ &.,.$\leqslant १-$ & $1 \varepsilon$ \\
\hline$\cdot$, Y^० & $1,7 \leq r$ & TI &.,$Y 70$ &., .91 & 10 \\
\hline$\cdot, Y V A$ & $1, I V r$ & rr & . זา9 & .,V9r- & 17 \\
\hline
\end{tabular}

وقد تمَّ حساب المُتوسِّط الحسـابي والحد الأدنى والحد الأعلى لتقديرات معالم الفقرة كما في الجدول رقم(7)

جدول (7): المُتوسيّطط الحسابي الحد الأدنى والحد الأعلى لتقديرات معالم الفقرة

\begin{tabular}{|c|c|c|c|}
\hline , rOA & الحد الأدنى للخطأ & $r,\{r \mid-$ & الحد الأدنى للصعوبة \\
\hline , ror & الحد الأعلى للخطأ & $r, \Lambda T \varepsilon$ & والحد الأعلى للصعوبة \\
\hline , YNT & المُمتوسِِطَط الحسابي & . & المُمَتُوِِّط الحسابي \\
\hline
\end{tabular}

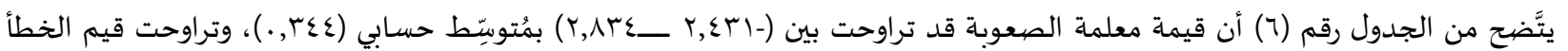

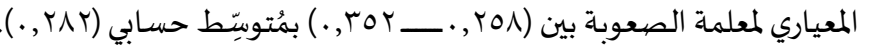

السُّؤال الرَّابع:ما درجة القطع لنموذج الاختباربصهورته النِّهائيَّة؟

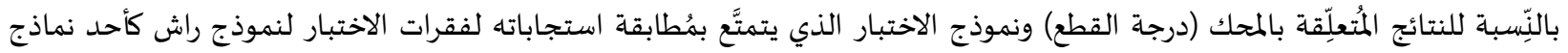

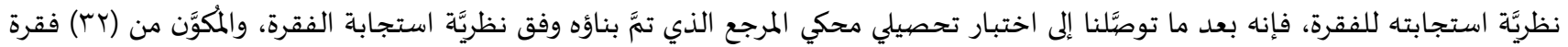

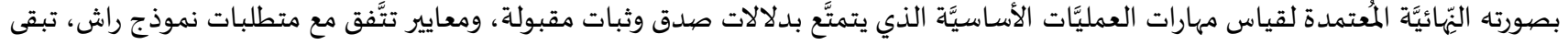

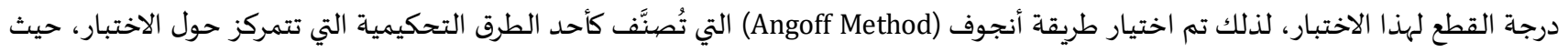

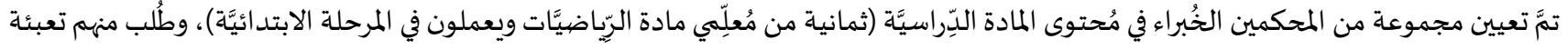
استبانة تقدير درجة القطع. ومن خلال اتِبّاع خطوات تحديد درجة القطع وفق طريقة أنجوف تمَّ تحديد درجة القطع التي قُدِّرت بـ(Y) درجهة، والجدول رقم (V) يُوضِّح ذلك.

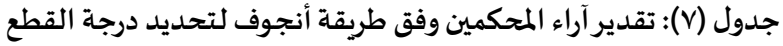

\begin{tabular}{|c|c|c|c|c|c|c|c|c|}
\hline المَّمَكِمَ & المُكَّكِمِم & السَّادسِ & الخُامسكِم & المُحكِّمِم الرَّابع & المُّكَكِمَ & المُّكَكِمَ & الأمُحكِكِم & أرقام الفقرات \\
\hline., 9 & $\cdot, \wedge$ & $\cdot, \wedge$ &., 9 & $\cdot, 9$ & $\cdot, \Lambda$ & $\cdot, 9$ & $\cdot, \Lambda$ & $(1$ \\
\hline ., 1 &., 9 & , , &.,$V$ & . 7 & , & , 10 & . 7 & (r \\
\hline.,$\Lambda$ & $\cdot, \wedge$ & $\cdot, \lambda$ &., 9 & $\cdot, \Lambda$ &., 9 &., 9 & $\cdot, \Lambda$ & $(r$ \\
\hline., $\mathrm{V}$ & $\cdot, \wedge$ & $\cdot, \wedge$ & ., & $\cdot, \mathrm{V}$ & $\cdot, \mathrm{V}$ &., 9 & $\cdot, \mathrm{V}$ & $(\varepsilon$ \\
\hline., 0 &., 0 & ., & ., $\mathrm{V}$ &., 7 &., 0 &., 0 & . 7 & 10 \\
\hline., 70 &., $\mathrm{~V}$ & $\cdot, \Lambda$ &., 9 &.,$\Lambda$ & $\cdot, 7$ &., 7 & $\cdot, \mathrm{V}$ & (7 \\
\hline ., & $\cdot, \wedge$ & ., Vo & ., V & ., & $\cdot, \Lambda$ & $\cdot, \mathrm{V}$ &., 9 & (V \\
\hline
\end{tabular}




\begin{tabular}{|c|c|c|c|c|c|c|c|c|}
\hline., $\mathrm{V}$ & $\cdot, \mathrm{V}$ & $\cdot, \mathrm{V}$ &., 9 & $\cdot, \mathrm{V}$ & $\cdot, 7$ & $\cdot, \wedge$ & $\cdot v$ & $(\Lambda$ \\
\hline .,V & . 7 &, 70 & ., & ., & ., & . 7 & ., & (9 \\
\hline ., & 7, & ., V & 7, & ., & 7, & ., & 7, & $(1$. \\
\hline$\cdot, 7$ &., 0 & $\cdot, \mathrm{V}$ &., 7 &., 0 &., 7 &., 7 &., 7 & $(11$ \\
\hline$\cdot, \mathrm{V}$ & ., Vo & $\cdot, \mathrm{V}$ & $\cdot, \wedge$ & $\cdot, \wedge$ &., 7 &., 9 &., 10 & (Ir \\
\hline$\cdot, \wedge$ & .,V & $\cdot, \wedge$ & •, V & $\cdot, \wedge$ & r, &., 9 &.,$\wedge$ & $(1 \pi$ \\
\hline$\cdot, \mathrm{V}$ & $\cdot, \mathrm{V}$ & $\cdot, \mathrm{V}$ &.,$\wedge$ & $\cdot, \wedge$ &., 7 & $\cdot, \mathrm{V}$ & $\cdot, \Lambda$ & $(1 \varepsilon$ \\
\hline$\cdot, \mathrm{V}$ & $\cdot, \mathrm{V}$ & $\cdot, 9$ & $\cdot, \mathrm{V}$ & $\cdot, \wedge$ & $\cdot, \wedge$ & $\cdot, \mathrm{V}$ &., 9 & $(10$ \\
\hline$\cdot, \mathrm{V}$ &., 9 &.,$\wedge$ &.,$\wedge$ &., 9 &.,$\wedge$ & $\cdot, \wedge$ &., 9 & $(17$ \\
\hline$\cdot, 9$ &., 9 &., 9 & $\cdot, \wedge$ &.,$\Lambda$ &., 9 & $\cdot, \wedge$ &., 9 & (IV \\
\hline., 7 &., 0 &., 0 &., 0 &., 7 & $\cdot, \mathrm{V}$ &, 00 & .0 & $(11$ \\
\hline., 0 &., 0 &., 0 &., 0 &., 7 & .7 &., 0 &., 0 & $(19$ \\
\hline$\cdot, \mathrm{V}$ & $\cdot, \mathrm{V}$ &., $\mathrm{V}$ & $\cdot, \wedge$ & $\cdot, \wedge$ &., 10 &., 7 & $\cdot, \wedge$ & $(r$. \\
\hline., 90 &., 90 & $\cdot, 9$ &., 9 & $\cdot, 9$ & $\cdot, 9$ & $\cdot, 9$ & $\cdot, 9$ & $(r)$ \\
\hline$\cdot, 7$ &., 10 & $\cdot, 7$ & $\cdot, 7$ & $\cdot, 7$ & $\cdot, \wedge$ & $\cdot, \mathrm{V}$ &., 0 & $(T Y$ \\
\hline$\cdot, 7$ & $\cdot, 7$ & $\cdot, 7$ & $\cdot, 7$ & $\cdot, 7$ & $\cdot, \wedge$ &., 7 &., 0 & $(T \pi$ \\
\hline$\cdot, 7$ &., 7 & $\cdot, 7$ & $\cdot, 7$ & $\cdot, 7$ & $\cdot, \wedge$ & $\cdot, 7$ &., 0 & $(T \varepsilon$ \\
\hline., 7 &., 7 & .7 &., 7 &., 7 &., 0 &., 7 &., 7 & (ro \\
\hline$\cdot, 7$ & $\cdot, 7$ & $\cdot, 7$ & $\cdot 7$ & $\cdot, 7$ & $\cdot, \wedge$ &., 70 & $\cdot, 7$ & $(r 7$ \\
\hline$\cdot, 7$ & $\cdot, 9$ & $\cdot, \wedge$ & $\cdot, \mathrm{V}$ & $\cdot, 7$ & $\cdot, \wedge$ & $\cdot, \mathrm{V}$ & $\cdot, \wedge$ & $(T V$ \\
\hline$\cdot, \wedge$ &., 7 & $\cdot, 10$ & $\cdot, \wedge$ & $\cdot, 7$ & $\cdot, \wedge$ & $\cdot, \wedge$ & $\cdot, \wedge$ & $(T \Lambda$ \\
\hline$\cdot, \wedge$ & $\cdot, \wedge$ & $\cdot, \wedge$ & $\cdot, \wedge$ &., 7 & $\cdot, \wedge$ & $\cdot, 7$ & $\cdot, \lambda$ & $(r 9$ \\
\hline$\cdot, 7$ &., 7 &., 7 &., 7 & $\cdot, 7$ &., 0 &., 7 & $\cdot, \mathrm{V}$ & $(r$. \\
\hline$\cdot, \mathrm{V}$ & $\cdot, 7$ &., 0 &., 7 & $\cdot, 7$ &., 0 & $\cdot, \wedge$ &., 7 & $(r)$ \\
\hline$\cdot, \mathrm{V}$ &., 0 &., 0 &., 0 &., 0 &., 0 &., 0 &., 00 & (rt \\
\hline Tr & YI & Tr & Tr & Tr & $r \mu$ & $r$ & Tr & المجموع \\
\hline
\end{tabular}

السُؤوال الخامس: ما مُستوى إتقان خرِّجِات المرحلة الابتدائيَّة لمهارات العمليَّات الحسابيَّة الأساسيَّة في مادة الرِِّّاضيات (الجمع، والطَّح،

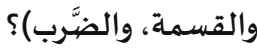

للإجابة عن هذا السُّؤال تمَّ حساب الدَّرجات الكلية لكل طالبة بالاختبار، وكذلك حساب درجاتها في كل مهارة من مهارات العمليَّات الحسابية

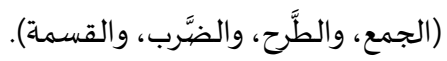

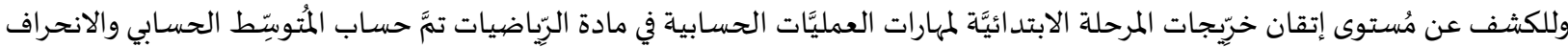

المعياري لدرجات الطالباًت الكلية على الاختبار كما في الجدول رقم (م). جدول (^): المُتُوبِّط الحسابي والانحراف المعياري لدرجات الطالباًت الكلية على الاختبار

\begin{tabular}{|c|c|c|c|}
\hline \multirow[t]{2}{*}{ الكلي } & حجم العِيِّة & المُتوسِِّط الحسابي & الانحراف المعياري \\
\hline & r.o & $|V,| \Lambda$ & $r, \leq 0$. \\
\hline
\end{tabular}

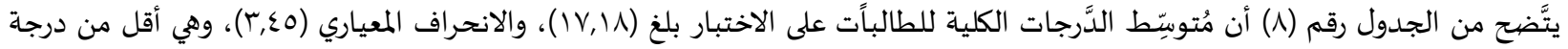

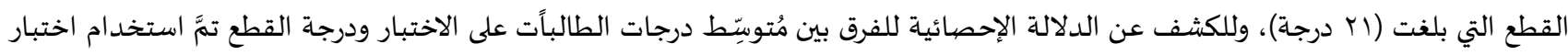
(T) والنَّائج كما في الجدول رقم (9) (9)

جدول (9): اختبار(T)لحساب الفرق بين مُتوسِّط درجات الطالباًت على الاختبارودرجة القطع

\begin{tabular}{|c|c|c|c|c|}
\hline \multirow[t]{2}{*}{ الكلي } & قيمة ت & درجات الحرية & مُستوى الدلالة & مُتوسِّط الفروق \\
\hline & 10,101 & T. $\varepsilon$ &.,. & \\
\hline
\end{tabular}

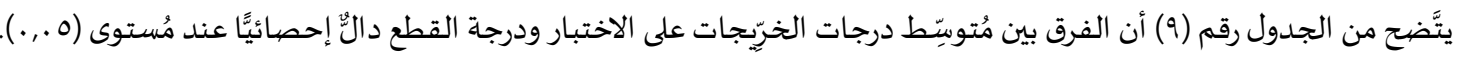


وللكشف عن مُستوى إتقان خرِّبجات المرحلة الابتدائَيَّة في كل مهارة :

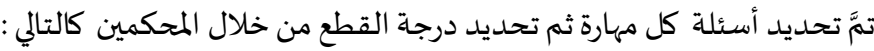

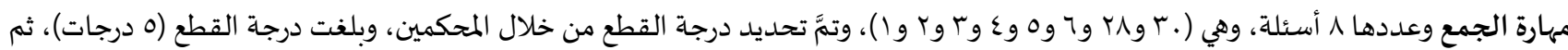

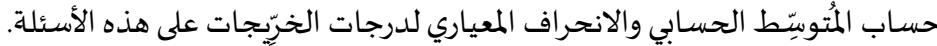

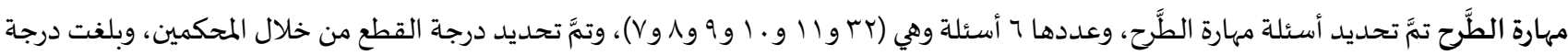
القطع (ع درجات)، ثم حسـاب المُتِوبِّط الحسابي والانحراف المعياري لدرجات الخرِِّجات على هذه الأسئلة.

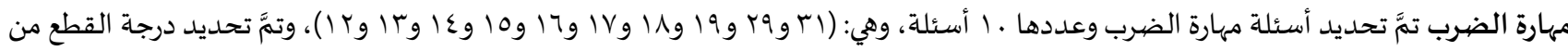

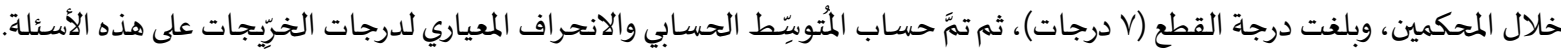

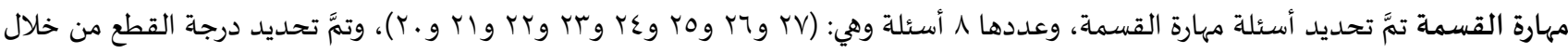

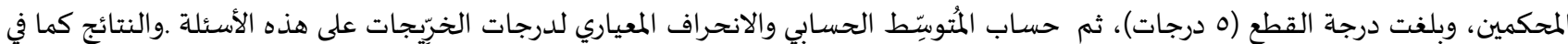

الجدول رقم (1) (1).

جدول ( ـ (): المُتُوِِّط الحسابي والانحراف المعياري لدرجات الخرِّجات على كل مهارة :

\begin{tabular}{|c|c|c|c|}
\hline مهارة & حجم العينة & المُتُوبِّط الحسابي & الانحراف المعياري \\
\hline الجمع & r.o & $0,1 \pi$ &., $9 \wedge \varepsilon$ \\
\hline الطرح & r.o & $r, \wedge 9$ & . $94 \pi$ \\
\hline الضرب & r.o & $\varepsilon, q 1$ & 1,207 \\
\hline القسمة & r.O & T, TO & $1, \varepsilon T r$ \\
\hline
\end{tabular}

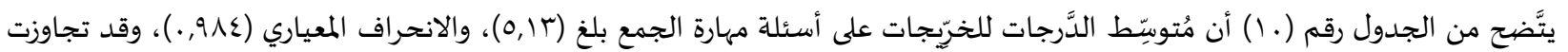

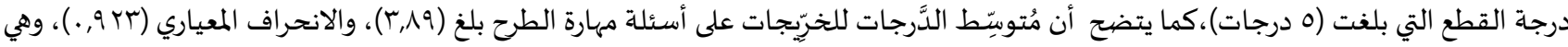

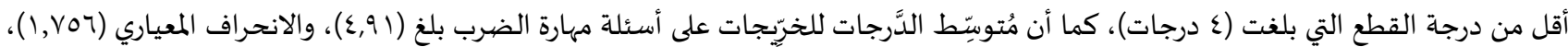

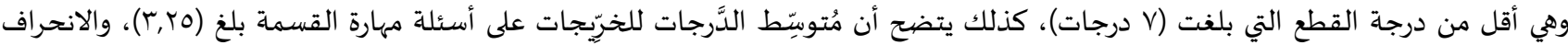

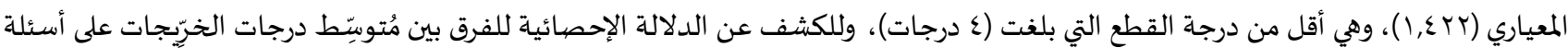

مهارات العمليات الأسـاسية ودرجة القطع لكل مهارة تمَّ استخدام اختبار (T)، والنَّتائج كما في الجدول رقيم (11).

جدول (1): اختبار( (T) لحسـاب للفرق بين مُتوبِّط درجات الخربجات على أسئلة مهارات العمليات الأسساسية ودرجة القطع

\begin{tabular}{|c|c|c|c|c|}
\hline المهارة & قيمة T T T & درجات الحرية & مستوى الدلالة & متوسط الفروق \\
\hline الجمع & $1,19 \mathrm{~V}$ & r. $\varepsilon$ &., $.0 \mathrm{~V}$ & . IrT \\
\hline الطرح & 1,770 & T.乏 &., $.9 \mathrm{~V}$ &., $1 \cdot V$ \\
\hline الضرب & $I V, .7$. & $r \cdot \varepsilon$ & $\cdot, .$. & $r, .9 T$ \\
\hline القسمة & $I V, 7 T V$ & $T \cdot \varepsilon$ & $\cdot, \ldots$ & 1,101 \\
\hline
\end{tabular}

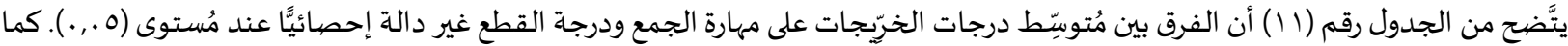

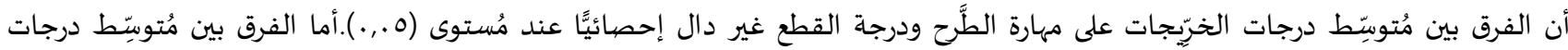

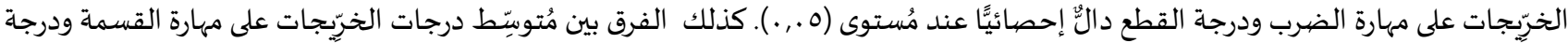

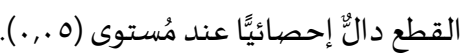
مُلْخَّص النَّتائج وتفسيرها:

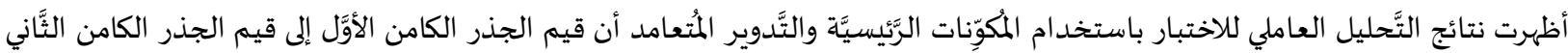

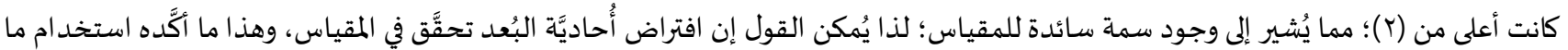

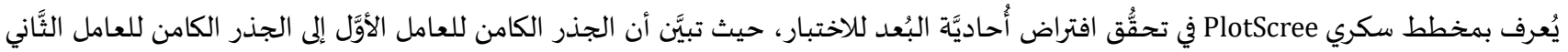
كانت عالية. 


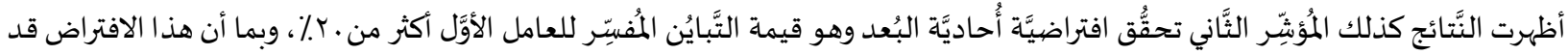

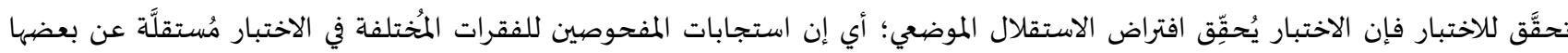

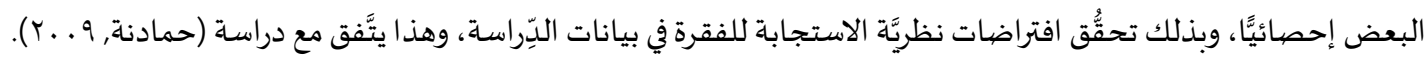

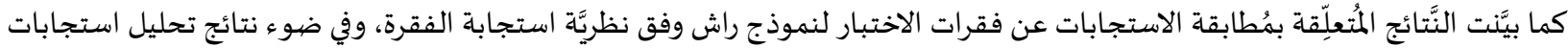

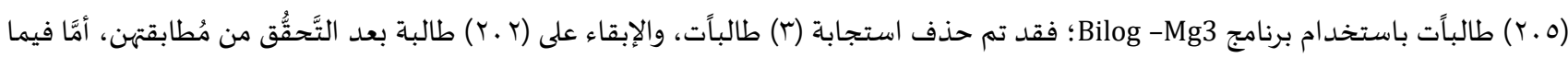

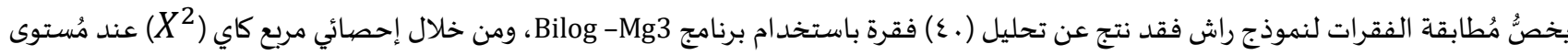

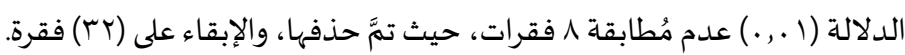

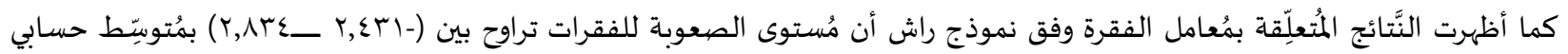

أمَّا فيما يتعلَّق بنتائج تحديد درجة القطع للاختبار فقد حُبِّدت درجة القطع بواسطة ثمانية مُحكِّمين وفق طريقة أنجوف، حيث قُدِّرت درجة القطع ب(ا) (Y) درجة.

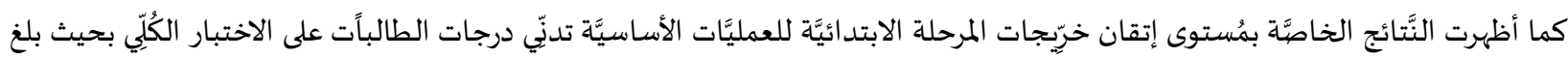

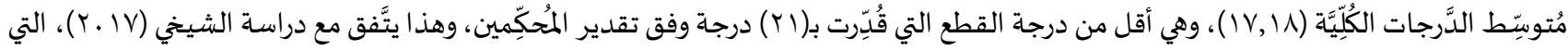

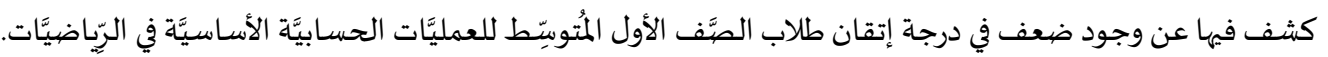

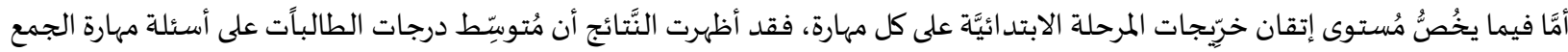

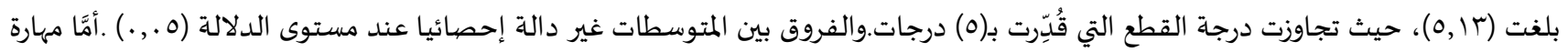

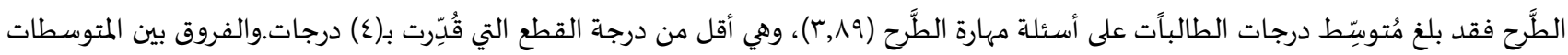

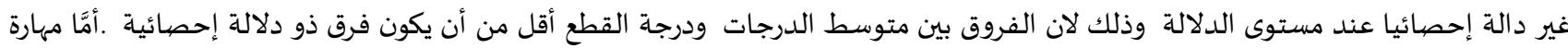

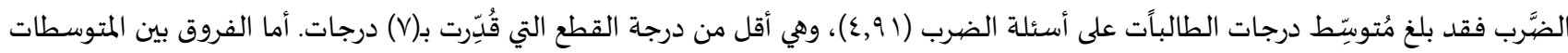

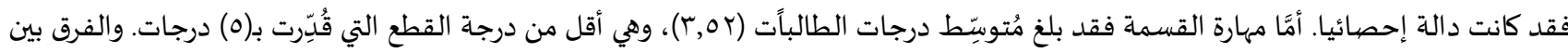

المتوسطات ذات دلالة إحصائية. التَّوصيَّات:

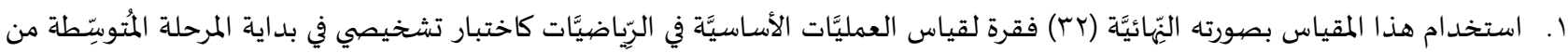
قِبِل مُعِلِّي الرِِّاضِيَّات.

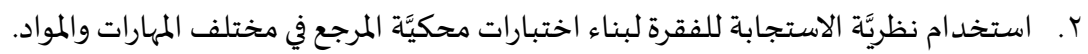

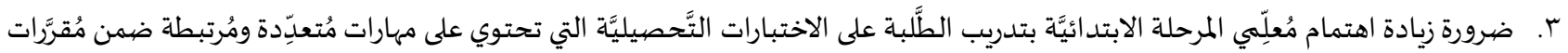

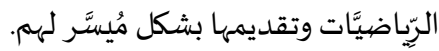

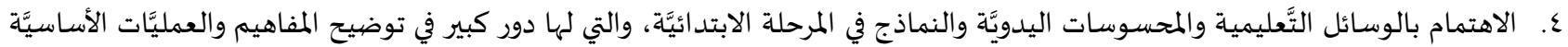
الرِّيَاضيَّة للطلبة.

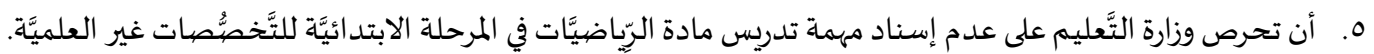
المُقترحات:

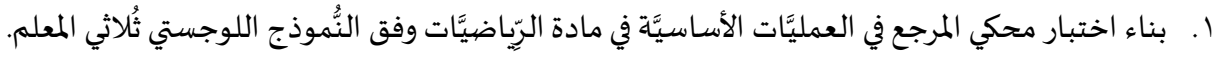

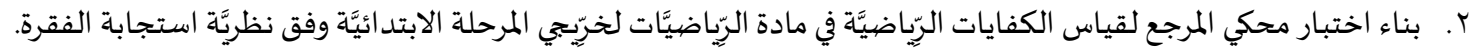

1. الحمادنة، أياد. (9 . .ب). "استخدام نظرية الاستجابة للمفردة في بناء اختبار محكي المرجع في الرياضيات وفق النموذج اللوجستي ثلاثي المعلم".

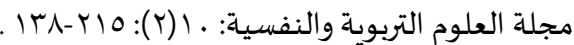
r. الختاتنة، رقية (ع (ب). "بناء اختبار محكمي المرجع في النسب المثلثية للصف التاسع الأساسي باستخدام نموذج راش". رسالة ماجستير غير منشورة. جامعة مؤتة. الأردن. 
r. الخياط، ماجد. (r ا . Y). "درجة مطابقة اختبار تحصيلي وفق نموذج راش أحادي المعلمة في الكشف عن مستوى المعرفة العلمية في المهارات

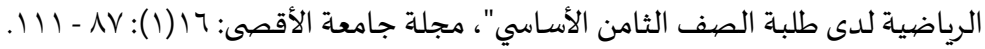

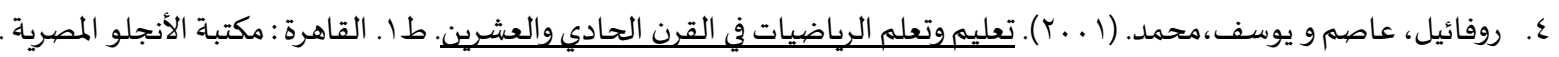

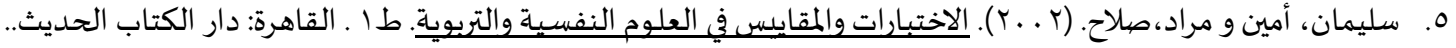

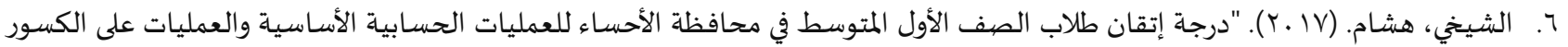

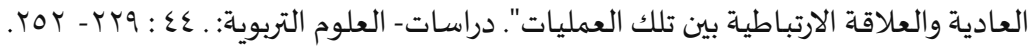

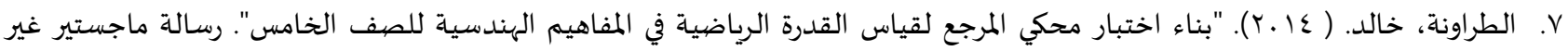
منشورة.جامعة مؤتة. الأردن.

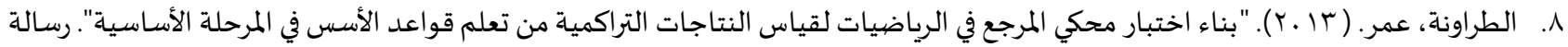
ماجستير غير منشورة.جامعة مؤتة. الأردن.

9. جامعة مؤتة. الأردن.

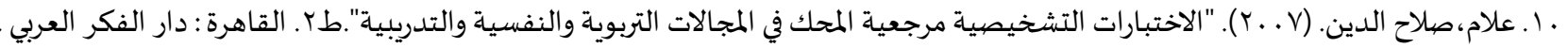

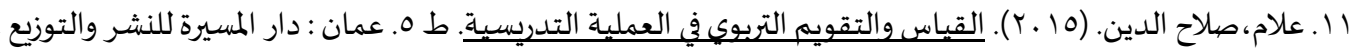
r ا . علي، عبدالكريم. (ا . . r). "القدرة الرياضية وعلاقتها بالتحصيل لدى طلبة الثانوية بالجمهورية اليمنية". رسالة ماجستير غير منشورة.جامعاة عدن. اليمن.

rا العنزي، عبدالعزيز؛ النذير، محمد. (7 (ب). "درجة اكتساب تلاميذ الصف السادس الابتدائي المعرفة الرياضية الأساسية المضيمنة مقرر

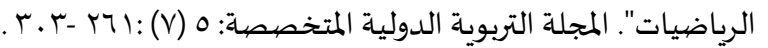
ع ا. عودة، أحمد. (ع ا . r ). القياس والتقويم في العملية التدريسية. ط ع. اربد : دار الأمل للنشر والتوزيع

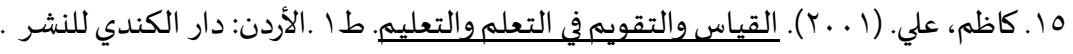

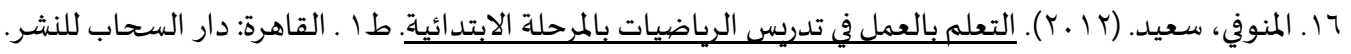

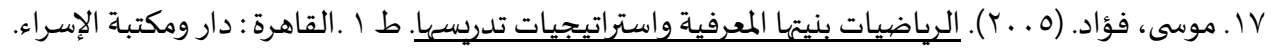

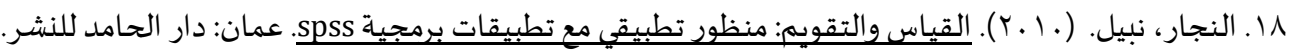

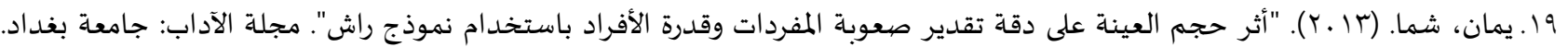
$.791 .-7 V \Gamma:(1.0)$

ثانياً: المراجع الأجنبية:

[1] Wilson, T, M \& Mac Gillivray (2007). "counting on the basics: Mathematical skills among tertiary entrants", International Journal of Mathematical Education in Science \& Technology.38(1): 19-41. https://doi.org/10.1080/00207390600819029.

[2] Hambleton, R., \& Swaminathan, H. (1985). "Item Response Theory: Principles and applications”, Boston: Kluwer Nijhoff Publishing. 
المجلة الدولية للدراسـات التربوية والنفسية

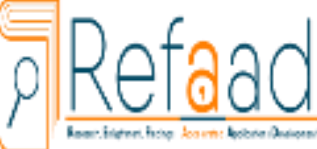

www.refaad.com
International Journal of Educational \& Psychological Studies (EPS)

Journal Homepage: https://www.refaad.com/views/EPSR/Home.aspx

ISSN: 2520-4149 (Online) 2520-4130 (Print)

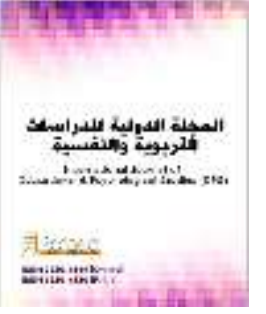

\title{
Construction of a reference test to measure basic mathematical processes of female primary graduates using response theory
}

\author{
Aisha Mohammed Sail Al-enazi \\ Researcher, KSA
}

Sabri Mohammed Ismail Abdel Aal

Assistant Professor in Educational Measurement and Evaluation, College of Education and Arts, University of Tabuk, KSA

sabdelaal@ut.edu.sa

\author{
Received : 31/4/2020 Revised : 10/4/2020 Accepted : 10/5/2020 DOI : https://doi.org/10.31559/EPS2021.9.1.11
}

Abstract: The aim of this study was to construct a reference test to measure the basic processes in mathematics according to the theory of paragraph response, where using the single- logistic model (Rush model), The study sample consisted of (205) students of the sixth-grade primary school in the city of Tabuk. To achieve the objective of the study, the test of the reference theorem was built in the basic operations of mathematics; consisting of 32 paragraphs of the type of multiple choices, the degree of cutting was determined to be (21), the results were analyzed by ITMAN, Bilog-MG3, and Spss. The results showed that the assumptions of the single-parameter logistic model were met (32) a paragraph, (8) non-conforming items were deleted, and the results of the sample included the exclusion of (3) individuals who did not conform to the model. The results of the analysis of female students on the overall test showed a low level of female students, where the average score (17.18), which is less than the degree of finalized, estimated at (21).Further the results of the analysis of the grades of the students on each skill showed that the average grade of the students was less than the finalized grade in all skills except for the skill of collection, which averaged the students' scores in the questions of the skill of collection $(5,13)$, Where it exceeded the degree of finalized, which was estimated at (5) degrees, In light of the results of the study, the researcher recommended using this measure in its final form to measure the basic processes in mathematics as a diagnostic test at the beginning of the intermediate stage by the mathematics teachers, She also recommended the need to increase the interest of primary school teachers in the training of students on the achievement tests that contain multiple skills and linked to the mathematics courses and provide them in a way that facilitates them.

Keywords: To construct a test; reference theorem; Basic Operations; Paragraph Response Theory.

\section{References:}

[1] Al'badlh, Smyh. (2014). "Bna' Akhtbar Mhky Almrj' Fy Whdh Thlyl Almqadyr Aljbryh Llsf Altas' Alasasy". Rsalt Majstyr Ghyr Mnshwrh. Jam't M'th. Alardn.

[2] 'lam,Slah Aldyn. (2007). "Alakhtbarat Altshkhysyh Mrj'yh Almhk Fy Almjalat Altrbwyh Walnfsyh Waltdrybyh". T2. Alqahrh : Dar Alfkr Al'erby .

[3] 'lam,Slah Aldyn. (2015). Alqyas Waltqwym Altrbwy Fy Al'mlyh Altdrysyh. T 5. 'man: Dar Almsyrh Llnshr Waltwzy'.

[4] 'ly, 'bdalkrym. (2001). "Alqdrh Alryadyh W'laqtha Balthsyl Lda Tlbt Althanwyh Baljmhwryh Alymnyh". Rsalt Majstyr Ghyr Mnshwrh. Jam't 'dn. Alymn.

[5] Al'nzy, 'bdal'zyz: Alndyr, Mhmd. (2016). "Drjt Aktsab Tlamyd Alsf Alsads Alabtda'y Alm'rfh Alryadyh Alasasyh Almdmnh Mqrr Alryadyat". Almjlh Altrbwyh Aldwlyh Almtkhssh: 5 (7) :261 -303. 
[6] 'wdh, Ahmd. (2014). Alqyas Waltqwym Fy Al'mlyh Altdrysyh. T 4. Arbd: Dar Alaml Llnshr Waltwzy'.

[7] Alhmadnh, Ayad. (2009). "Astkhdam Nzryh Alastjabh Llmfrdh Fy Bna' Akhtbar Mhky Almrj' Fy Alryadyat Wfq Alnmwdj Allwjsty Thlathy Alm'lm". Mjlt Al'lwm Altrbwyh Walnfsyh: 10(2): 215-138.

[8] Alkhtatnh, Rqyh (2014). "Bna' Akhtbar Mhkmy Almrj' Fy Alnsb Almthlthyh Llsf Altas' Alasasy Bastkhdam Nmwdj Rash". Rsalt Majstyr Ghyr Mnshwrh. Jam't M'th. Alardn.

[9] Kazm, 'ly. (2001). Alqyas Waltqwym Fy Alt'Im Walt'lym. T1. Alardn: Dar Alkndy Llnshr .

[10] Alkhyat, Majd. (2012). "Drjt Mtabqh Akhtbar Thsyly Wfq Nmwdj Rash Ahady Alm'lmh Fy Alkshf 'n Mstwa Alm'rfh Al'lmyh Fy Almharat Alryadyh Lda Tlbt Alsf Althamn Alasasy", Mjlt Jam't Alaqsa: 16(1): 87 - 111.

[11] Almnwfy, S'yd. (2012). Alt'lm Bal'ml Fy Tdrys Alryadyat Balmrhlh Alabtda'yh. T1. Alqahrh: Dar Alshab Llnshr.

[12] Mwsa, F'ad. (2005). Alryadyat Bnytha Alm'rfyh Wastratyjyat Tdrysha. T 1. Alqahrh : Dar Wmktbh Alesra'.

[13] Alnjar, Nbyl. (2010). Alqyas Waltqwym: Mnzwr Ttbyqy M' Ttbyqat Brmjyh Spss. 'man: Dar Alhamd Llnshr.

[14] Rwfa'yl, 'Easm W Ywsf,Mhmd. (2001). T'lym Wt'lm Alryadyat Fy Alqrn Alhady Wal'eshryn. T1. Alqahrh: Mktbt Alanjlw Almsryh .

[15] Slyman, Amyn W Mrad,Slah. (2002). Alakhtbarat Walmqayys Fy Al'lwm Alnfsyh Waltrbwyh. T1. Alqahrh: Dar Alktab Alhdyth.

[16] Alshykhy, Hsham. (2017). "Drjt Etqan Tlab Alsf Alawl Almtwst Fy Mhafzt Alahsa' Ll'mlyat Alhsabyh Alasasyh Wal'mlyat 'la Alkswr Al'adyh Wal'laqh Alartbatyh Byn Tlk Al'mlyat". Drasat- Al'elwm Altrbwyh: 44: 229- 252.

[17] Altrawnh, Khald. (2014). "Bna' Akhtbar Mhky Almrj' Lqyas Alqdrh Alryadyh Fy Almfahym Alhndsyh Llsf Alkhams". Rsalt Majstyr Ghyr Mnshwrh. Jam't M'th. Alardn.

[18] Altrawnh, 'mr. (2013). "Bna' Akhtbar Mhky Almrj' Fy Alryadyat Lqyas Alntajat Altrakmyh Mn T'lm Qwa'd Alass Fy Almrhlh Alasasyh". Rsalt Majstyr Ghyr Mnshwrh. Jam't M'th. Alardn.

[19] Yman, Shma. (2013). "Athr Hjm Al'ynh 'la Dqh Tqdyr S'ewbh Almfrdat Wqdrh Alafrad Bastkhdam Nmwdj Rash". Mjlt Aladab: Jam't Bghdad. (105): 673 - 6980. 